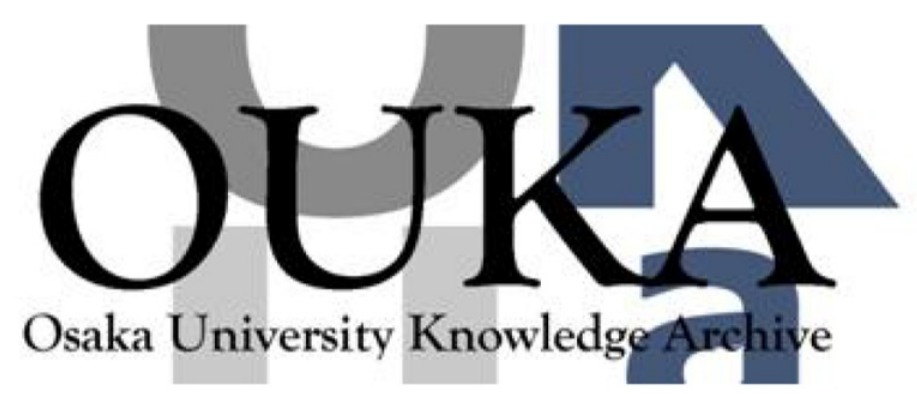

\begin{tabular}{|c|c|}
\hline Title & $\begin{array}{l}\text { Frequency dependence of images in scanning } \\
\text { laser source technique for a plate }\end{array}$ \\
\hline Author (s) & $\begin{array}{l}\text { Hayashi, Takahiro; Murase, Morimasa; Kitayama, } \\
\text { Tsunaji }\end{array}$ \\
\hline Citation & Ultrasonics. 52(5) p. 636-p. 642 \\
\hline Issue Date & $2012-07$ \\
\hline oaire:version & AM \\
\hline URL & https://hdl. handle. net/11094/84497 \\
\hline rights & $\begin{array}{l}\text { - } 2012 \text { Elsevier B.V. This manuscript version is } \\
\text { made avai lable under the Creative Commons } \\
\text { Attribut ion-NonCommercial-NoDerivatives } 4.0 \\
\text { International License. }\end{array}$ \\
\hline Note & \\
\hline
\end{tabular}

Osaka University Knowledge Archive : OUKA

https://ir. Library. osaka-u. ac. jp/

Osaka University 


\title{
Frequency dependence of images in scanning laser source technique for a plate
}

Takahiro Hayashi, Morimasa Murase, Tsunaji Kitayama

Toyota Central R\&D Labs., Inc., Nagakute, Aichi, 480-1192, Japan

\begin{abstract}
Defect imaging using scanning laser source technique has been investigated for a plate with rounded defects and notch-type defects in our previous studies. This paper examines frequency dependence of the defect images with both calculations and experiments in order to acquire clearer images. Both calculation and experimental results for a straight notch revealed that clearer images of notch-type defects can be obtained in the range of low frequency-thickness product below about $200 \mathrm{kHz} \cdot \mathrm{mm}$. Moreover, images of the defects of various shapes were obtained by synthesizing images from 8 receiving transducers, and similarly to the case of the straight notches, they became more clearly in the low frequency range.
\end{abstract}

PACS numbers: 43.35.Zc, 43.34.YB, and 43.20.Mv

Keywords: Nondestructive testing, Laser ultrasonics, Lamb waves, Imaging

Contact author: Takahiro Hayashi: Phone: +81-561-71-7485, Fax: +81-561-63-6859,

e-mail: takahiro-hayashi@mosk.tytlabs.co.jp 


\section{Introduction}

Laser ultrasonic technique ${ }^{[1]}$ is highly attracted as a non-contact inspection technique, in which ultrasonic is emitted by thermoelastic stress or ablation effect induced by laser pulse emission onto the surface of a material and ultrasonic is received by interferometry between a reference beam and a reflected or scattered beam from the surface.

Thus considering inspection of the existing structures and products in manufacturing lines, the reflected or scattered laser beam cannot be stably received due to surface conditions such as roughness, reflection angle and vibration, even if ultrasonic emission is feasible. As a solution of such a problem in on-line inspections, we took note of scanning laser source (SLS) technique in which ultrasonic emission is done by a pulse laser and ultrasonic is received with ultrasonic transducers fixed on a surface of the material.

The SLS technique provides a wide variety of information on materials from many waveforms acquired when laser beam is scanned over the material with mirrors. Kromine et. al. ${ }^{[2]}$, Fomitchov et. al ${ }^{[3]}$, , and Shon et.al ${ }^{[4]}$ developed the SLS for surface breaking cracks using amplitude and frequency changes of waveforms. Takatsubo et.al. ${ }^{[5]}$ proposed defect detection technique where the animation of wave propagation is created from the waveforms acquired by the SLS and defect were detected from distortions of the wave propagation. Authors ${ }^{[6],[7]}$ reported that amplitude distributions of the A0 mode of Lamb waves in the frequency range below the A1 cut-off frequency roughly correspond to thickness distributions for a plate with a rounded shallow defect. Moreover, images of notch-type defects were also obtained by synthesizing plural images from plural receivers ${ }^{[8]}$.

In this paper, frequency dependences of synthesized images using plural receivers in the SLS are investigated with both calculations and experiments for a plate with notch-type defects. The outline of defect imaging by the SLS with plural receivers for a plate with notch-type defects is described first, and the frequency dependences of the amplitude distributions are analyzed by calculations, and finally the frequency dependences of images are investigated by SLS experiments. 


\section{Outline of defect imaging with scanning laser source with plural receivers}

Characteristics of A0 mode of Lamb waves are utilized in the defect imaging technique with the SLS for a plate. Fig.1 is a schematic figure of signals received at the fixed transducer when laser beam is emitted onto the surface of a plate with a rounded defect. Large signals are detected when the laser beam is emitted on the thin defected region (Fig.1(b)), while detected signals become small when laser beam is on the thick intact regions (Fig.1 (a), (c)). As a result, an amplitude distribution can be obtained by scanning the laser source as shown in Fig.1 (d). These phenomena appear when reflection is small at a defect, and our previous pape ${ }^{[6]}$ demonstrated that thickness distribution images can be obtained when A0 mode of Lamb waves from the SLS is detected in the low frequency region below the A1 cut-off frequency for a shallow rounded defect.

However, for defects that generate large reflected waves such as notches and deep defects, the amplitude distributions do not correspond to the thickness distributions even using the A0 mode in the low frequency range. Fig.2 (a) shows a schematic figure of an amplitude distribution around a notch-type defect. Wavy amplitude distribution at the transducer side is caused by interferences of direct and reflected waves, and it results in the spurious images in two dimensional amplitude distributions (defect images). Thus, our previous work ${ }^{[8]}$ introduced imaging technique using plural receivers where defect images are enhanced by taking summation or product of plural images. Fig.2 (b) is a schematic figure of the synthesizing process of two amplitude distributions. The two upper distributions show amplitude distributions measured at receiving points 1 and 2 . These two amplitude distributions commonly have large values at the defect area, while have different distributions at the intact regions. Taking summation or product of these two amplitude distributions gives enhanced amplitude distributions where the defect area has large value and the other intact areas become small.

Although the amplitude distribution measured by the SLS corresponds to thickness distribution in a case of small reflection ${ }^{[6]}$, the amplitude distribution affected by large reflection as shown in Fig.2 (a) has no physical meaning. Therefore, the synthesizing process using summation or product of plural images is just an enhancement process of defect images, and one can choose an effective means in each measurement. In this study, we use the synthesizing technique by summation. 


\section{Theoretical investigation on frequency dependence of amplitude distributions}

In the previous work ${ }^{[6]}$, amplitude distributions were calculated for rounded defects of various shapes by the combined model of a semi-analytical finite element (SAFE) method and a finite element (FE) method ${ }^{[9],[10]}$. In this section, using the same calculation model, amplitude distributions are calculated for a plate with a notch-type defect.

Now, we consider an aluminum plate (longitudinal wave velocity $c_{L}=6260 \mathrm{~m} / \mathrm{s}$, transverse wave velocity $c_{T}=3080 \mathrm{~m} / \mathrm{s}$ ) of thickness $d$ having a rectangular defect of width $w$ and depth $h$ as shown in Fig.3. The SAFE regions on the both side of the plate model are divided into 16 layers, and the FE region with the length of $20 d$ between the SAFE regions is divided into a $16 \times 160$ rectangular mesh. A harmonic vibration of frequency $f$ in the thickness direction is applied at FE nodes on the upper surface from $x=-9.875 d$ to $x=+9.875 d$, and then normal displacements of A0 mode on the upper surface of the right SAFE region are calculated. Since attenuation of ultrasonic is not considered in the calculation, the amplitude of the normal displacement of a single A0 mode is constant at any places in the right SAFE region.

In the calculation described above, the loading points of harmonic vibration $x$ and the receiving point of the normal displacement of A0 mode correspond to laser source points and a location of a receiving transducer in the SLS, respectively. Amplitude distributions are calculated from receiving signals at a single receiver or dual receivers for various loading points. Note that this calculation does not strictly express ultrasonic generation by laser in the experiments discussed below. In the experiments, ultrasonic is generated in the thermoelastic regime where in-plane stresses are mainly generated. However, since many parameters have to be considered for the precise model of laser generation such as laser spot size, surface condition of a material, and pulse energy of the laser ${ }^{[1],[4]}$, this paper uses normal loading condition for qualitative analyses of A0 mode with out-of-plane displacement.

In theoretical studies and calculations of Lamb waves, a frequency and thickness product $f d$ is used instead of frequency $f$ for generalization, and all lengths are often represented by the ratio to thickness $d$. This paper also uses the notation, $f d, w / d, h / d, x / d$, and temporally calls them frequency, width, depth and location, respectively.

\subsection{Amplitude distributions around a notch with a single receiver}


Fig.4 shows the amplitude distributions (loading point $x / d$ versus normalized amplitude) around the notch of width $w / d=0.5$ and depth $h / d=0.5$ for various frequencies, $f d=40,200,500,1000$, $2000 \mathrm{kHz} \cdot \mathrm{mm}$ in a single receiver located at the right SAFE region. The amplitude distributions are totally different in the left and right regions with respect to the notch center due to the reflection at the notch region shown by the dashed lines in Fig.4. In the right region of the notch, the same side of the receiving transducer as loading points, amplitude change periodically appears due to the interference of direct waves and reflected waves from the notch. On the contrary, at the left loading points, amplitude becomes small due to small transmitted waves at the notch. Similar amplitude change was observed at the vicinity of a surface breaking crack $^{[2-4]}$ due to the interference, but for a plate with a notch as in this study, periodical amplitude changes can be seen even in the far field from the notch about $x / d>5$.

Though the amplitude distributions are inversely proportional to the thickness distributions for shallow rounded defects as in Fig.1(d), large distortions of amplitude distribution are observed in defects where large reflected waves occur. The previous work ${ }^{[6]}$ described that such reflected waves put limitations on thickness measurements using the SLS.

\subsection{Synthesized amplitude distributions around a notch with dual receivers}

The large distortions of amplitude distribution were experimentally observed for notch-type defects. In the previous study ${ }^{[8]}$, in order to reduce spurious images caused by such distortions and to enhance defect images, a synthesized image were created by taking summation or product of plural images from plural receivers as shown in Fig.2.

Applying the image enhancement technique to amplitude distribution with a single receiver of Fig.4 gives the distributions of Fig.5. These amplitude distributions are summations of two distributions from dual receivers located at the right and left intact regions. The amplitude distributions became symmetry with respect to the notch center. Moreover, the maximum amplitudes were observed at the center of the notch for $f d=40$ and $200 \mathrm{kHz} \cdot \mathrm{mm}$, which implies that enhanced defect images can be obtained using multiple receivers. Since all images from multiple receivers have large values at defect center for $f d=40$ and $200 \mathrm{kHz} \cdot \mathrm{mm}$, taking the summation of these images enhances defect images. However, as spurious distributions still largely remain in it, this technique cannot be used for quantitative evaluation such as thickness measurement in shallow rounded 
defects ${ }^{[6]}$.

\subsection{Frequency dependence of imaging performance by dual receivers}

The summation of amplitude distributions from dual receivers in low frequency range gives large values at defect area as shown in Fig.5 (a) and (b), which were also experimentally verified ${ }^{[8]}$. In this section, therefore, imaging performance is evaluated using the imaging performance index $A / B$ which is calculated by $A$ and $B$ as shown in Fig.6.

Fig.6 is a zoomed graph of Fig.5 (a). Dashed lines in the figure denote the notch region of $-0.25<x / d<0.25$. Defining $A$ and $B$ are the maximum values inside and outside the notch region, respectively, larger imaging index $A / B$ means that clearer defect images can be observed as shown in Fig.5 (a) and (b). On the contrary, for small imaging index $A / B$ below 1 , wavy and noisy amplitude distributions are observed outside the notch as in Fig.5 (d) and (e).

Fig.7 shows distributions of the imaging index $A / B$ for the widths of $w / d=0.25$ and 0.5 in the graph of frequency $f d$ versus depth $h / d$. The values at $h / d=0.5$ in Fig.7 (b) correspond to the amplitude distributions in Fig.5. In both figures, imaging index $A / B$ indicates more than 1 (gray) in the low frequency range below $f d=200 \mathrm{kHz} \cdot \mathrm{mm}$, which means that large amplitudes are observed inside the notch region as shown in Fig.5 (a) and (b). On the other hand, imaging index $A / B$ is below 1 (white) in the frequency range of $f d=500-1400 \mathrm{kHz} \cdot \mathrm{mm}$ because largest amplitudes appear outside the notch region as seen in Fig.5 (c) and (d).

Very large $A / B$ values are seen at the right upper corner of Fig.7 (b), which may be caused by resonance in the notch region. However, considering the fact that the $A / B$ is below 1 for shallow notches $h / d<0.35$ or for narrow notches $w / d=0.25$ (Fig.7(a)) in the frequency range of $f d>1500$ $\mathrm{kHz} \cdot \mathrm{mm}$, defect images are strongly affected by notch depth and width in the frequency range, which implies that this imaging technique cannot be applied to unknown defects in this high frequency range $f d>1500 \mathrm{kHz} \cdot \mathrm{mm}$.

\section{Experimental investigation on frequency dependence of defect images}

In order to evaluate the imaging performance experimentally, defect images are acquired using the experimental system shown in Fig.8 and an aluminum test plate shown in Fig.9 The experimental system consists of a pulse laser equipment (A Q-switched YAG laser), a galvano mirror 
scanner, and ultrasonic receivers. Broadband pin-type transducers with a small diameter (Valpey Fisher, VP-1093, 2.3mm diameter, nominal frequency range DC-1.2MHz) were adopted as the receiving transducers. Laser beam was scanned over the plate with the galvano mirror scanner, and then amplitude distribution images were acquired for all receiving transducers. Finally, a synthesized image was obtained by taking the summation of the amplitude distribution images ${ }^{[8]}$.

Notch-type defects of different shapes and different depth were engraved on the back surface of the aluminum plate as shown in Fig.9. The defect shapes were straight lines (A), zigzag patterns (B), large T-shape (C), and small T-shape (D) from the left row to the right, and the notch depths vary from the upper column to the bottom at $0.5,0.3,0.2$, and $0.1 \mathrm{~mm}$.

\subsection{Results for straight notch defects}

Firstly, defect imaging experiments were carried out for straight notches shown in Fig.9(A) to compare calculation results. As shown in Fig.10, dual ultrasonic transducers were located at the both side about $20 \mathrm{~mm}$ away from the notch center. Fig. 11 shows defect images $(10 \times 23 \mathrm{~mm}, 0.5 \mathrm{~mm}$ steps) of straight notches with four different depths and in 8 different frequency ranges. The 8 frequency ranges were provided using band-pass filters with cut-off frequencies shown in the Fig.11.

Clearer images of the straight notches can be seen for deeper notches and in lower frequency ranges in Fig.11, which agrees well with calculation results in Fig.7 (b) where the imaging index $A / B$ became larger in the low frequency ranges below $f d=200 \mathrm{kHz} \cdot \mathrm{mm}$ and for deeper notches.

Some other experimental results also agree well with calculation ones. For example, edge images are emphasized for $h=0.5 \mathrm{~mm}$ and in the frequency range of $400-500 \mathrm{kHz}$, which can be seen in the calculation result of Fig.5 (c). At the frequency above $500 \mathrm{kHz}$, periodical longitudinal lines were observed on both sides of the notch, especially for $h=0.5 \mathrm{~mm}$, which also agrees with Fig.5 (d) and (e).

\subsection{Results for various defects}

Imaging experiments for defects of various shapes A-D were carried out using eight receiving transducers placed at the corners and the center of sides, as shown in Fig.8. The SLS was scanned over the region of $150 \times 220 \mathrm{~mm}$ in steps of $1 \mathrm{~mm}$, and synthesized images were obtained in four different frequency ranges as shown in Fig.12. Darker dots represent large synthesized values, and distinct defect images were obtained for the straight notch defects (A), zigzag defects (B), and 
large T-shaped defects (C).

Looking at the change of these defect images for various frequencies $f d$ and depths $h / d$, clearer images were obtained for deeper defects and in the lower frequency range. And the shallowest defects of $h / d=0.1$ cannot be detected in the high frequency ranges of (c) and (d). These tendencies agree well with the calculation results in Fig.7 and the experimental results for straight notches shown in Fig.11.

In Fig.12 (b)-(d), longitudinal, transverse and diagonal stripes can be seen in addition to the defect images. These stripes are due to interferences between direct waves and reflected waves from the plate edges.

The small T-shaped defects (D) were not detected in the all images, because amplitude change did not occur due to diffraction around the small defects. The diffraction was not considered in the above-mentioned calculation. Three dimensional calculation of Lamb wave propagation is required for detailed analyses of the diffraction.

\section{Conclusions}

This paper investigated the frequency dependence of images in the SLS with calculations and experiments. The combined model of SAFE and FE methods was used for calculating synthesized amplitude distributions of A0 mode by the SLS for a plate with a notch-type defect. The calculation results indicated that clearer images can be obtained below about $200 \mathrm{kHz} \cdot \mathrm{mm}$ of frequency-thickness product, which agreed well with experimental results for straight notches. Similarly, in the experiments with 8 receiving transducers, the defects of various shapes appeared in the synthesized images more clearly in the low frequency range.

The images in the SLS are also affected by the number and locations of receiving transducers, the extent and shape of defects, surface conditions of the material and so on. Their further studies may be required for practical use.

\section{References}

[1] C. B. Scruby, L. E. Drain, Laser ultrasonics: Techniques and Applications, Adam Hilger, New York, 1990. 
[2] A. Kromine, P. Fomitchov, Sridhar Krishnaswamy, and J. D. Achenbach, Laser ultrasonic detection of surface breaking discontinuities: Scanning laser source technique, Mater. Eval.. 58 (2) (2000) 173.

[3] P. A. Fomitchov, A. K. Kromine, Y. Sohn, S. Krishnaswamy, and J. D. Achenbach, Ultrasonic imaging of small surface-breaking defects using scanning laser source technique, in D. Thompson, D. Chimenti (Eds.), Review of Progress in Quantitative Nondestructive Evaluation, American Institute of Physics, 2002, pp.356-362.

[4] Y. Sohn and S. Krishnaswamy, "Interaction of a scanning laser-generated ultrasonic line source with a surface-breaking flaw", J. Acoust. Soc. Am., 115 (1), (2004) 172.

[5] J. Takatsubo, B. Wang, H. Tsuda, N. Tooyama, Generation laser scanning method for the visualization of ultrasounds propagating on a 3-D object with an arbitrary shape, J. Solid Mechanics and Materials Eng., 1 (12), (2007) 1405.

[6] T. Hayashi, M. Murase, and M. N. Salim, Rapid thickness measurements using guided waves from a scanning laser source, J. Acoust. Soc. Am., 126 (3), (2009) 1101.

[7] M. N. Salim, T. Hayashi, M. Murase, T. Ito, and S. Kamiya, Laser based measurements of plate thickness using a fundamental antisymmetric mode of lamb wave, in D. Thompson, D. Chimenti (Eds.), Review of Progress in Quantitative Nondestructive Evaluation, American Institute of Physics, 2010, pp.231-238.

[8] T. Hayashi, M. Murase, and T. Kitayama, Defect imaging technique using a scanning laser source, in D. Thompson, D. Chimenti (Eds.), Review of Progress in Quantitative Nondestructive Evaluation, American Institute of Physics, 2011, pp.713-719.

[9] T. Hayashi, W.-J. Song and J. L. Rose, Guided wave dispersion curves for a bar with an arbitrary cross-section, a rod and rail example Ultrasonics, 41 (3) (2003) 175.

[10] T. Hayashi and J. L. Rose, Guided wave simulation and visualization by a semi-analytical finite element method, Mater. Eval., 61, (2003) 75. 


\title{
Frequency dependence of images in scanning laser source technique for a plate
}

Takahiro Hayashi, Morimasa Murase, Tsunaji Kitayama

Toyota Central R\&D Labs., Inc., Nagakute, Aichi, 480-1192, Japan

\begin{abstract}
Defect imaging using scanning laser source technique has been investigated for a plate with rounded defects and notch-type defects in our previous studies. This paper examines frequency dependence of the defect images with both calculations and experiments in order to acquire clearer images. Both calculation and experimental results for a straight notch revealed that clearer images of notch-type defects can be obtained in the range of low frequency-thickness product below about $200 \mathrm{kHz} \cdot \mathrm{mm}$. Moreover, images of the defects of various shapes were obtained by synthesizing images from 8 receiving transducers, and similarly to the case of the straight notches, they became more clearly in the low frequency range.
\end{abstract}

PACS numbers: 43.35.Zc, 43.34.YB, and 43.20.Mv

Keywords: Nondestructive testing, Laser ultrasonics, Lamb waves, Imaging

Contact author: Takahiro Hayashi: Phone: +81-561-71-7485, Fax: +81-561-63-6859, e-mail: takahiro-hayashi@mosk.tytlabs.co.jp 


\section{Introduction}

Laser ultrasonic technique ${ }^{[1]}$ is highly attracted as a non-contact inspection technique, in which ultrasonic is emitted by thermoelastic stress or ablation effect induced by laser pulse emission onto the surface of a material and ultrasonic is received by interferometry between a reference beam and a reflected or scattered beam from the surface.

Thus considering inspection of the existing structures and products in manufacturing lines, the reflected or scattered laser beam cannot be stably received due to surface conditions such as roughness, reflection angle and vibration, even if ultrasonic emission is feasible. As a solution of such a problem in on-line inspections, we took note of scanning laser source (SLS) technique in which ultrasonic emission is done by a pulse laser and ultrasonic is received with ultrasonic transducers fixed on a surface of the material.

The SLS technique provides a wide variety of information on materials from many waveforms acquired when laser beam is scanned over the material with mirrors. Kromine et. al. ${ }^{[2]}$, Fomitchov et. al ${ }^{[3]}$, , and Shon et.al ${ }^{[4]}$ developed the SLS for surface breaking cracks using amplitude and frequency changes of waveforms. Takatsubo et.al. ${ }^{[5]}$ proposed defect detection technique where the animation of wave propagation is created from the waveforms acquired by the SLS and defect were detected from distortions of the wave propagation. Authors ${ }^{[6],[7]}$ reported that amplitude distributions of the A0 mode of Lamb waves in the frequency range below the A1 cut-off frequency roughly correspond to thickness distributions for a plate with a rounded shallow defect. Moreover, images of notch-type defects were also obtained by synthesizing plural images from plural receivers ${ }^{[8]}$.

In this paper, frequency dependences of synthesized images using plural receivers in the SLS are investigated with both calculations and experiments for a plate with notch-type defects. The outline of defect imaging by the SLS with plural receivers for a plate with notch-type defects is described first, and the frequency dependences of the amplitude distributions are analyzed by calculations, and finally the frequency dependences of images are investigated by SLS experiments. 


\section{Outline of defect imaging with scanning laser source with plural receivers}

Characteristics of A0 mode of Lamb waves are utilized in the defect imaging technique with the SLS for a plate. Fig.1 is a schematic figure of signals received at the fixed transducer when laser beam is emitted onto the surface of a plate with a rounded defect. Large signals are detected when the laser beam is emitted on the thin defected region (Fig.1(b)), while detected signals become small when laser beam is on the thick intact regions (Fig.1 (a), (c)). As a result, an amplitude distribution can be obtained by scanning the laser source as shown in Fig.1 (d). These phenomena appear when reflection is small at a defect, and our previous pape ${ }^{[6]}$ demonstrated that thickness distribution images can be obtained when A0 mode of Lamb waves from the SLS is detected in the low frequency region below the A1 cut-off frequency for a shallow rounded defect.

However, for defects that generate large reflected waves such as notches and deep defects, the amplitude distributions do not correspond to the thickness distributions even using the A0 mode in the low frequency range. Fig.2 (a) shows a schematic figure of an amplitude distribution around a notch-type defect. Wavy amplitude distribution at the transducer side is caused by interferences of direct and reflected waves, and it results in the spurious images in two dimensional amplitude distributions (defect images). Thus, our previous work ${ }^{[8]}$ introduced imaging technique using plural receivers where defect images are enhanced by taking summation or product of plural images. Fig.2 (b) is a schematic figure of the synthesizing process of two amplitude distributions. The two upper distributions show amplitude distributions measured at receiving points 1 and 2 . These two amplitude distributions commonly have large values at the defect area, while have different distributions at the intact regions. Taking summation or product of these two amplitude distributions gives enhanced amplitude distributions where the defect area has large value and the other intact areas become small.

Although the amplitude distribution measured by the SLS corresponds to thickness distribution in a case of small reflection ${ }^{[6]}$, the amplitude distribution affected by large reflection as shown in Fig.2 (a) has no physical meaning. Therefore, the synthesizing process using summation or product of plural images is just an enhancement process of defect images, and one can choose an effective means in each measurement. In this study, we use the synthesizing technique by summation. 


\section{Theoretical investigation on frequency dependence of amplitude distributions}

In the previous work ${ }^{[6]}$, amplitude distributions were calculated for rounded defects of various shapes by the combined model of a semi-analytical finite element (SAFE) method and a finite element (FE) method ${ }^{[9],[10]}$. In this section, using the same calculation model, amplitude distributions are calculated for a plate with a notch-type defect.

Now, we consider an aluminum plate (longitudinal wave velocity $c_{L}=6260 \mathrm{~m} / \mathrm{s}$, transverse wave velocity $c_{T}=3080 \mathrm{~m} / \mathrm{s}$ ) of thickness $d$ having a rectangular defect of width $w$ and depth $h$ as shown in Fig.3. The SAFE regions on the both side of the plate model are divided into 16 layers, and the FE region with the length of $20 d$ between the SAFE regions is divided into a $16 \times 160$ rectangular mesh. A harmonic vibration of frequency $f$ in the thickness direction is applied at FE nodes on the upper surface from $x=-9.875 d$ to $x=+9.875 d$, and then normal displacements of A0 mode on the upper surface of the right SAFE region are calculated. Since attenuation of ultrasonic is not considered in the calculation, the amplitude of the normal displacement of a single A0 mode is constant at any places in the right SAFE region.

In the calculation described above, the loading points of harmonic vibration $x$ and the receiving point of the normal displacement of A0 mode correspond to laser source points and a location of a receiving transducer in the SLS, respectively. Amplitude distributions are calculated from receiving signals at a single receiver or dual receivers for various loading points. Note that this calculation does not strictly express ultrasonic generation by laser in the experiments discussed below. In the experiments, ultrasonic is generated in the thermoelastic regime where in-plane stresses are mainly generated. However, since many parameters have to be considered for the precise model of laser generation such as laser spot size, surface condition of a material, and pulse energy of the laser ${ }^{[1],[4]}$, this paper uses normal loading condition for qualitative analyses of A0 mode with out-of-plane displacement.

In theoretical studies and calculations of Lamb waves, a frequency and thickness product $f d$ is used instead of frequency $f$ for generalization, and all lengths are often represented by the ratio to thickness $d$. This paper also uses the notation, $f d, w / d, h / d, x / d$, and temporally calls them frequency, width, depth and location, respectively.

\subsection{Amplitude distributions around a notch with a single receiver}


Fig.4 shows the amplitude distributions (loading point $x / d$ versus normalized amplitude) around the notch of width $w / d=0.5$ and depth $h / d=0.5$ for various frequencies, $f d=40,200,500,1000$, $2000 \mathrm{kHz} \cdot \mathrm{mm}$ in a single receiver located at the right SAFE region. The amplitude distributions are totally different in the left and right regions with respect to the notch center due to the reflection at the notch region shown by the dashed lines in Fig.4. In the right region of the notch, the same side of the receiving transducer as loading points, amplitude change periodically appears due to the interference of direct waves and reflected waves from the notch. On the contrary, at the left loading points, amplitude becomes small due to small transmitted waves at the notch. Similar amplitude change was observed at the vicinity of a surface breaking crack $^{[2-4]}$ due to the interference, but for a plate with a notch as in this study, periodical amplitude changes can be seen even in the far field from the notch about $x / d>5$.

Though the amplitude distributions are inversely proportional to the thickness distributions for shallow rounded defects as in Fig.1(d), large distortions of amplitude distribution are observed in defects where large reflected waves occur. The previous work ${ }^{[6]}$ described that such reflected waves put limitations on thickness measurements using the SLS.

\subsection{Synthesized amplitude distributions around a notch with dual receivers}

The large distortions of amplitude distribution were experimentally observed for notch-type defects. In the previous study ${ }^{[8]}$, in order to reduce spurious images caused by such distortions and to enhance defect images, a synthesized image were created by taking summation or product of plural images from plural receivers as shown in Fig.2.

Applying the image enhancement technique to amplitude distribution with a single receiver of Fig.4 gives the distributions of Fig.5. These amplitude distributions are summations of two distributions from dual receivers located at the right and left intact regions. The amplitude distributions became symmetry with respect to the notch center. Moreover, the maximum amplitudes were observed at the center of the notch for $f d=40$ and $200 \mathrm{kHz} \cdot \mathrm{mm}$, which implies that enhanced defect images can be obtained using multiple receivers. Since all images from multiple receivers have large values at defect center for $f d=40$ and $200 \mathrm{kHz} \cdot \mathrm{mm}$, taking the summation of these images enhances defect images. However, as spurious distributions still largely remain in it, this technique cannot be used for quantitative evaluation such as thickness measurement in shallow rounded 
defects ${ }^{[6]}$.

\subsection{Frequency dependence of imaging performance by dual receivers}

The summation of amplitude distributions from dual receivers in low frequency range gives large values at defect area as shown in Fig.5 (a) and (b), which were also experimentally verified ${ }^{[8]}$. In this section, therefore, imaging performance is evaluated using the imaging performance index $A / B$ which is calculated by $A$ and $B$ as shown in Fig.6.

Fig.6 is a zoomed graph of Fig.5 (a). Dashed lines in the figure denote the notch region of $-0.25<x / d<0.25$. Defining $A$ and $B$ are the maximum values inside and outside the notch region, respectively, larger imaging index $A / B$ means that clearer defect images can be observed as shown in Fig.5 (a) and (b). On the contrary, for small imaging index $A / B$ below 1 , wavy and noisy amplitude distributions are observed outside the notch as in Fig.5 (d) and (e).

Fig.7 shows distributions of the imaging index $A / B$ for the widths of $w / d=0.25$ and 0.5 in the graph of frequency $f d$ versus depth $h / d$. The values at $h / d=0.5$ in Fig.7 (b) correspond to the amplitude distributions in Fig.5. In both figures, imaging index $A / B$ indicates more than 1 (gray) in the low frequency range below $f d=200 \mathrm{kHz} \cdot \mathrm{mm}$, which means that large amplitudes are observed inside the notch region as shown in Fig.5 (a) and (b). On the other hand, imaging index $A / B$ is below 1 (white) in the frequency range of $f d=500-1400 \mathrm{kHz} \cdot \mathrm{mm}$ because largest amplitudes appear outside the notch region as seen in Fig.5 (c) and (d).

Very large $A / B$ values are seen at the right upper corner of Fig.7 (b), which may be caused by resonance in the notch region. However, considering the fact that the $A / B$ is below 1 for shallow notches $h / d<0.35$ or for narrow notches $w / d=0.25$ (Fig.7(a)) in the frequency range of $f d>1500$ $\mathrm{kHz} \cdot \mathrm{mm}$, defect images are strongly affected by notch depth and width in the frequency range, which implies that this imaging technique cannot be applied to unknown defects in this high frequency range $f d>1500 \mathrm{kHz} \cdot \mathrm{mm}$.

\section{Experimental investigation on frequency dependence of defect images}

In order to evaluate the imaging performance experimentally, defect images are acquired using the experimental system shown in Fig.8 and an aluminum test plate shown in Fig.9 The experimental system consists of a pulse laser equipment (A Q-switched YAG laser), a galvano mirror 
scanner, and ultrasonic receivers. Broadband pin-type transducers with a small diameter (Valpey Fisher, VP-1093, 2.3mm diameter, nominal frequency range DC-1.2MHz) were adopted as the receiving transducers. Laser beam was scanned over the plate with the galvano mirror scanner, and then amplitude distribution images were acquired for all receiving transducers. Finally, a synthesized image was obtained by taking the summation of the amplitude distribution images ${ }^{[8]}$.

Notch-type defects of different shapes and different depth were engraved on the back surface of the aluminum plate as shown in Fig.9. The defect shapes were straight lines (A), zigzag patterns (B), large T-shape (C), and small T-shape (D) from the left row to the right, and the notch depths vary from the upper column to the bottom at $0.5,0.3,0.2$, and $0.1 \mathrm{~mm}$.

\subsection{Results for straight notch defects}

Firstly, defect imaging experiments were carried out for straight notches shown in Fig.9(A) to compare calculation results. As shown in Fig.10, dual ultrasonic transducers were located at the both side about $20 \mathrm{~mm}$ away from the notch center. Fig. 11 shows defect images $(10 \times 23 \mathrm{~mm}, 0.5 \mathrm{~mm}$ steps) of straight notches with four different depths and in 8 different frequency ranges. The 8 frequency ranges were provided using band-pass filters with cut-off frequencies shown in the Fig.11.

Clearer images of the straight notches can be seen for deeper notches and in lower frequency ranges in Fig.11, which agrees well with calculation results in Fig.7 (b) where the imaging index $A / B$ became larger in the low frequency ranges below $f d=200 \mathrm{kHz} \cdot \mathrm{mm}$ and for deeper notches.

Some other experimental results also agree well with calculation ones. For example, edge images are emphasized for $h=0.5 \mathrm{~mm}$ and in the frequency range of $400-500 \mathrm{kHz}$, which can be seen in the calculation result of Fig.5 (c). At the frequency above $500 \mathrm{kHz}$, periodical longitudinal lines were observed on both sides of the notch, especially for $h=0.5 \mathrm{~mm}$, which also agrees with Fig.5 (d) and (e).

\subsection{Results for various defects}

Imaging experiments for defects of various shapes A-D were carried out using eight receiving transducers placed at the corners and the center of sides, as shown in Fig.8. The SLS was scanned over the region of $150 \times 220 \mathrm{~mm}$ in steps of $1 \mathrm{~mm}$, and synthesized images were obtained in four different frequency ranges as shown in Fig.12. Darker dots represent large synthesized values, and distinct defect images were obtained for the straight notch defects (A), zigzag defects (B), and 
large T-shaped defects (C).

Looking at the change of these defect images for various frequencies $f d$ and depths $h / d$, clearer images were obtained for deeper defects and in the lower frequency range. And the shallowest defects of $h / d=0.1$ cannot be detected in the high frequency ranges of (c) and (d). These tendencies agree well with the calculation results in Fig.7 and the experimental results for straight notches shown in Fig.11.

In Fig.12 (b)-(d), longitudinal, transverse and diagonal stripes can be seen in addition to the defect images. These stripes are due to interferences between direct waves and reflected waves from the plate edges.

The small T-shaped defects (D) were not detected in the all images, because amplitude change did not occur due to diffraction around the small defects. The diffraction was not considered in the above-mentioned calculation. Three dimensional calculation of Lamb wave propagation is required for detailed analyses of the diffraction.

\section{Conclusions}

This paper investigated the frequency dependence of images in the SLS with calculations and experiments. The combined model of SAFE and FE methods was used for calculating synthesized amplitude distributions of A0 mode by the SLS for a plate with a notch-type defect. The calculation results indicated that clearer images can be obtained below about $200 \mathrm{kHz} \cdot \mathrm{mm}$ of frequency-thickness product, which agreed well with experimental results for straight notches. Similarly, in the experiments with 8 receiving transducers, the defects of various shapes appeared in the synthesized images more clearly in the low frequency range.

The images in the SLS are also affected by the number and locations of receiving transducers, the extent and shape of defects, surface conditions of the material and so on. Their further studies may be required for practical use.

\section{References}

[1] C. B. Scruby, L. E. Drain, Laser ultrasonics: Techniques and Applications, Adam Hilger, New York, 1990. 
[2] A. Kromine, P. Fomitchov, Sridhar Krishnaswamy, and J. D. Achenbach, Laser ultrasonic detection of surface breaking discontinuities: Scanning laser source technique, Mater. Eval.. 58 (2) (2000) 173.

[3] P. A. Fomitchov, A. K. Kromine, Y. Sohn, S. Krishnaswamy, and J. D. Achenbach, Ultrasonic imaging of small surface-breaking defects using scanning laser source technique, in D. Thompson, D. Chimenti (Eds.), Review of Progress in Quantitative Nondestructive Evaluation, American Institute of Physics, 2002, pp.356-362.

[4] Y. Sohn and S. Krishnaswamy, "Interaction of a scanning laser-generated ultrasonic line source with a surface-breaking flaw", J. Acoust. Soc. Am., 115 (1), (2004) 172.

[5] J. Takatsubo, B. Wang, H. Tsuda, N. Tooyama, Generation laser scanning method for the visualization of ultrasounds propagating on a 3-D object with an arbitrary shape, J. Solid Mechanics and Materials Eng., 1 (12), (2007) 1405.

[6] T. Hayashi, M. Murase, and M. N. Salim, Rapid thickness measurements using guided waves from a scanning laser source, J. Acoust. Soc. Am., 126 (3), (2009) 1101.

[7] M. N. Salim, T. Hayashi, M. Murase, T. Ito, and S. Kamiya, Laser based measurements of plate thickness using a fundamental antisymmetric mode of lamb wave, in D. Thompson, D. Chimenti (Eds.), Review of Progress in Quantitative Nondestructive Evaluation, American Institute of Physics, 2010, pp.231-238.

[8] T. Hayashi, M. Murase, and T. Kitayama, Defect imaging technique using a scanning laser source, in D. Thompson, D. Chimenti (Eds.), Review of Progress in Quantitative Nondestructive Evaluation, American Institute of Physics, 2011, pp.713-719.

[9] T. Hayashi, W.-J. Song and J. L. Rose, Guided wave dispersion curves for a bar with an arbitrary cross-section, a rod and rail example Ultrasonics, 41 (3) (2003) 175.

[10] T. Hayashi and J. L. Rose, Guided wave simulation and visualization by a semi-analytical finite element method, Mater. Eval., 61, (2003) 75. 


\section{Figure 1}

Schematic figures representing the relationship between location of laser incidence and amplitude of receiving signals.
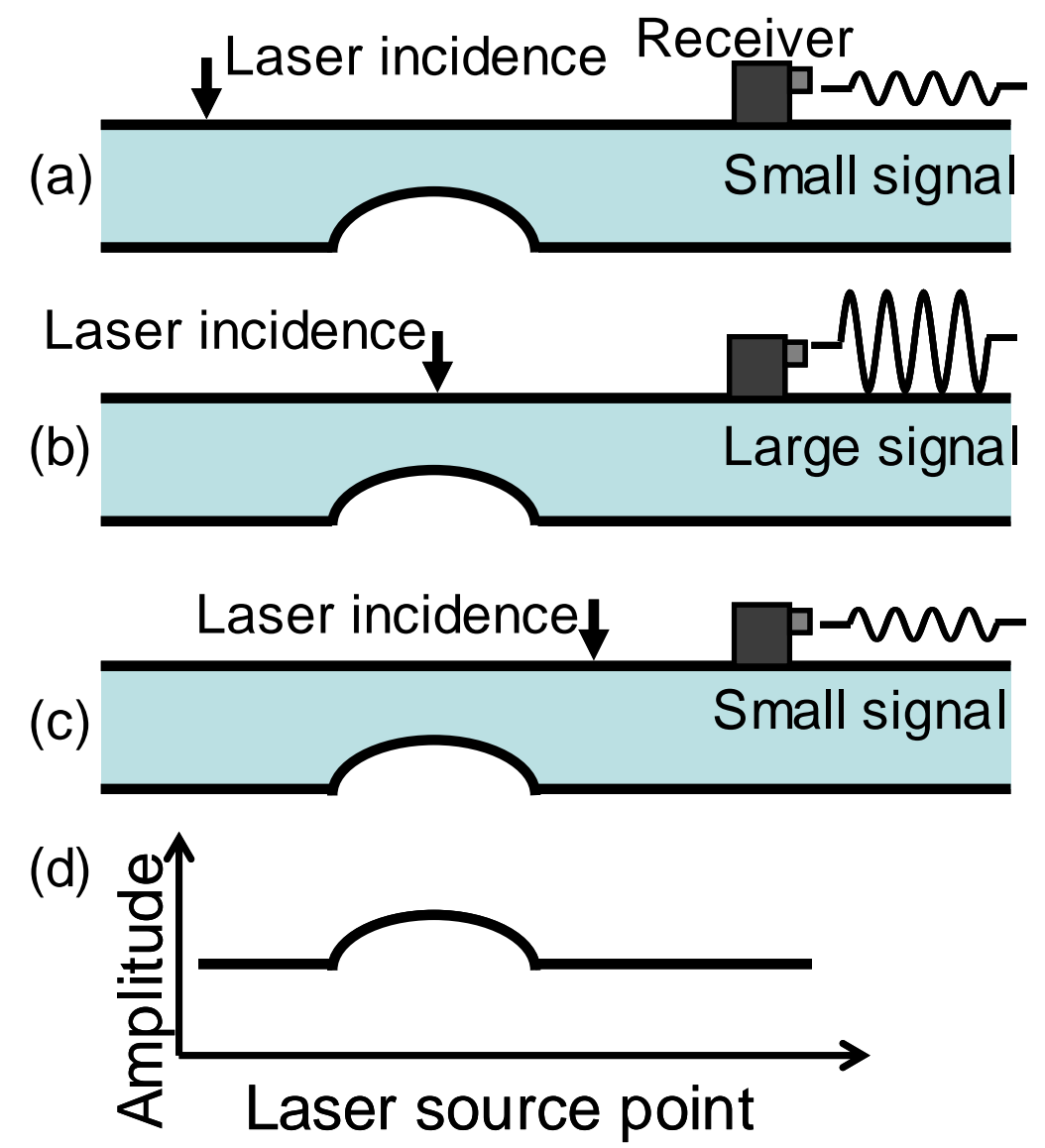


\section{Figure 2}

Schematic figures of amplitude distributions affected by large reflections and synthesized distributions using two receiving transducers.

(a)
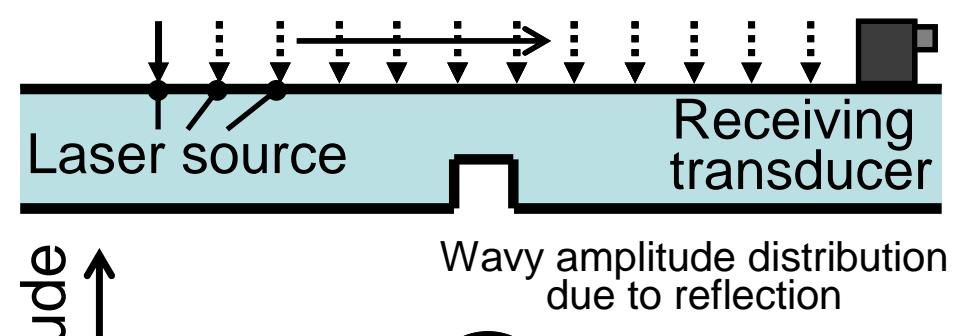

(b)

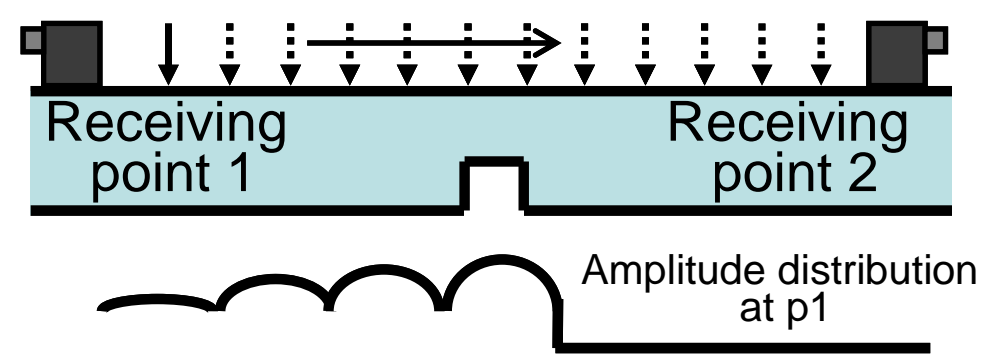

Amplitude distribution at p2

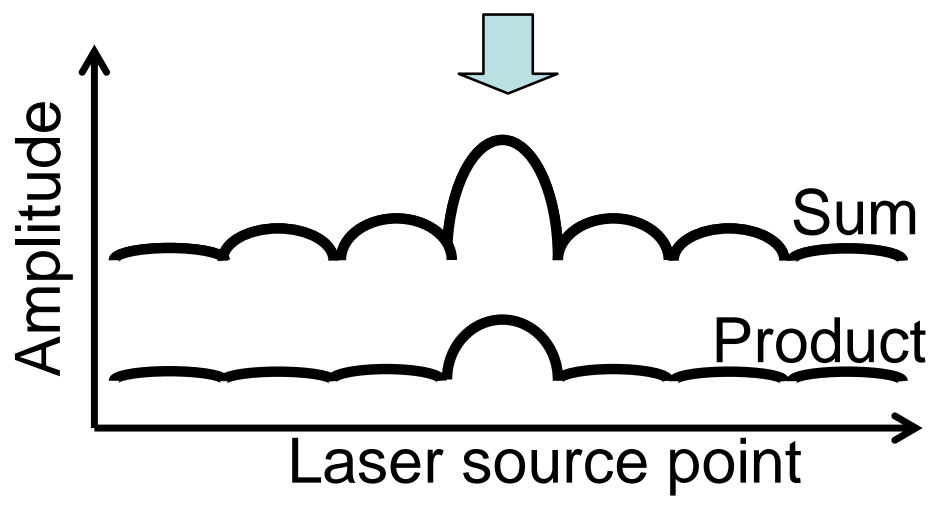




\section{Figure 3}

Combination model of SAFE and FE for calculating amplitude distributions.

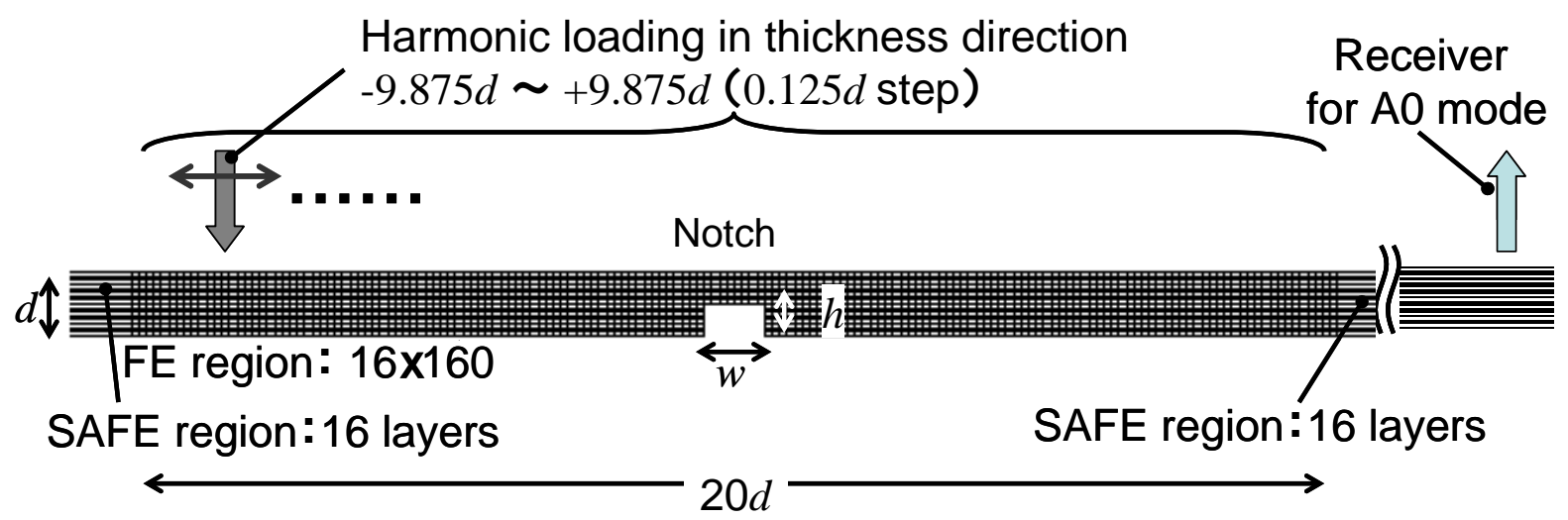


Figure 4

Amplitude distributions with a single receiver around a notch of $w / d=0.5$ and $h / d=0.5$ for various $f d$.

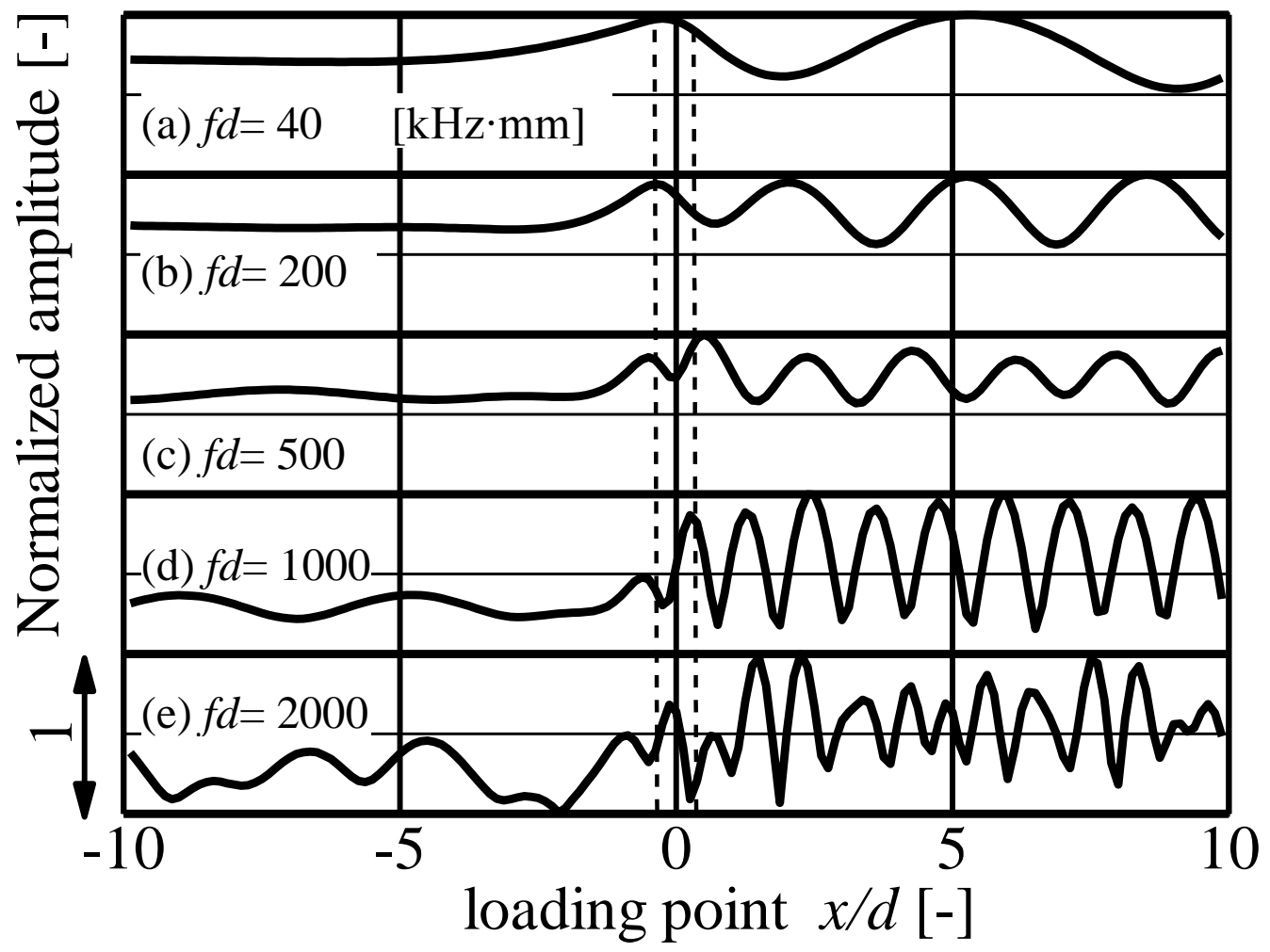


Figure 5

Summations of amplitude distributions with dual receivers located at both sides of a notch with $w / d=0.5$ and $h / d=0.5$ for various $f d$.

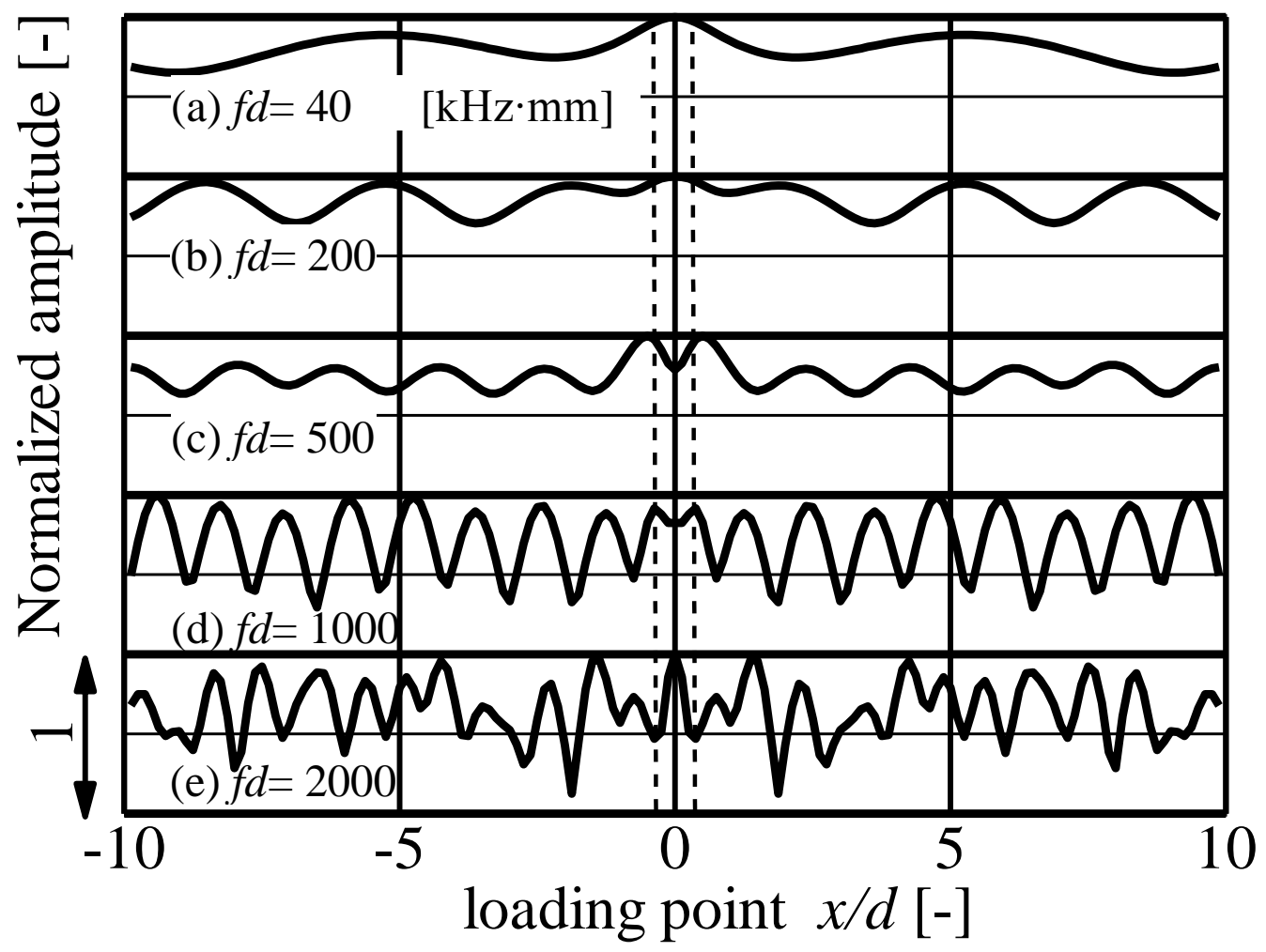


Figure 6

Definition of the imaging performance index $A / B$.

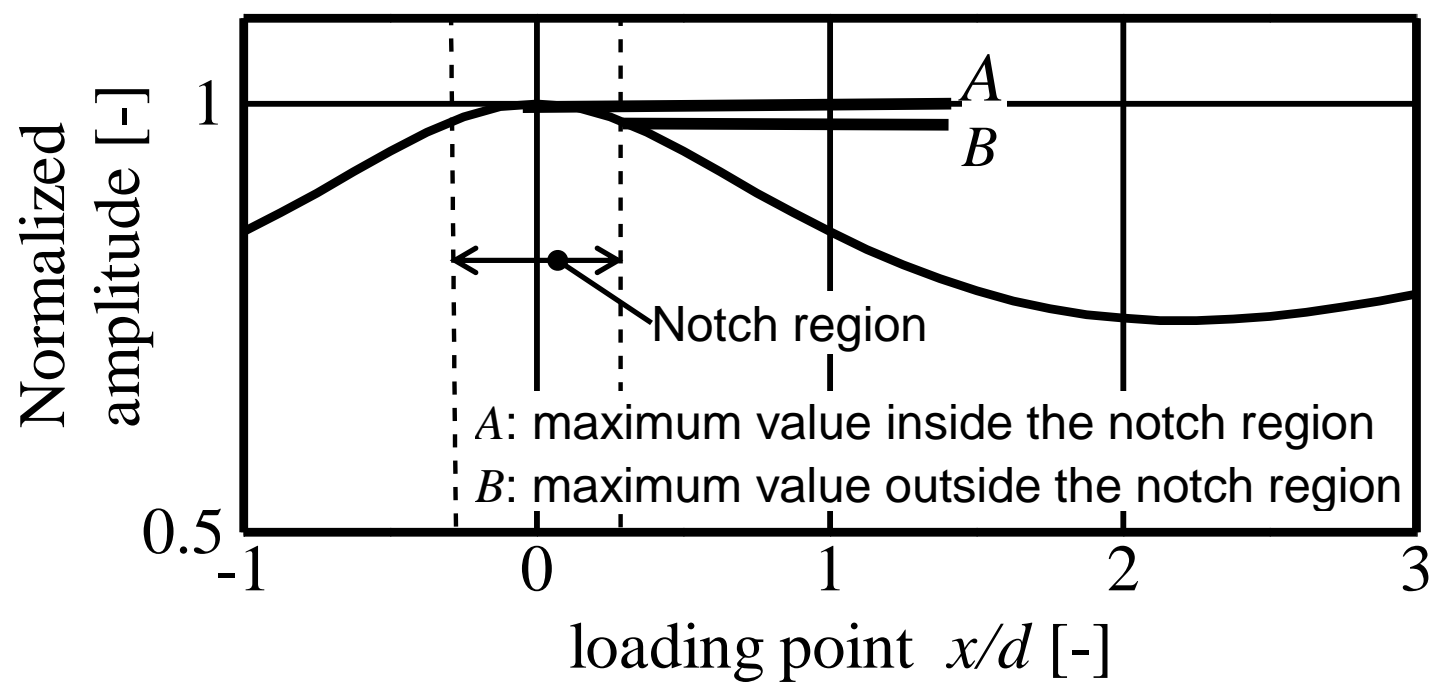




\section{Figure 7}

Frequency dependence of the imaging performance index $A / B$.

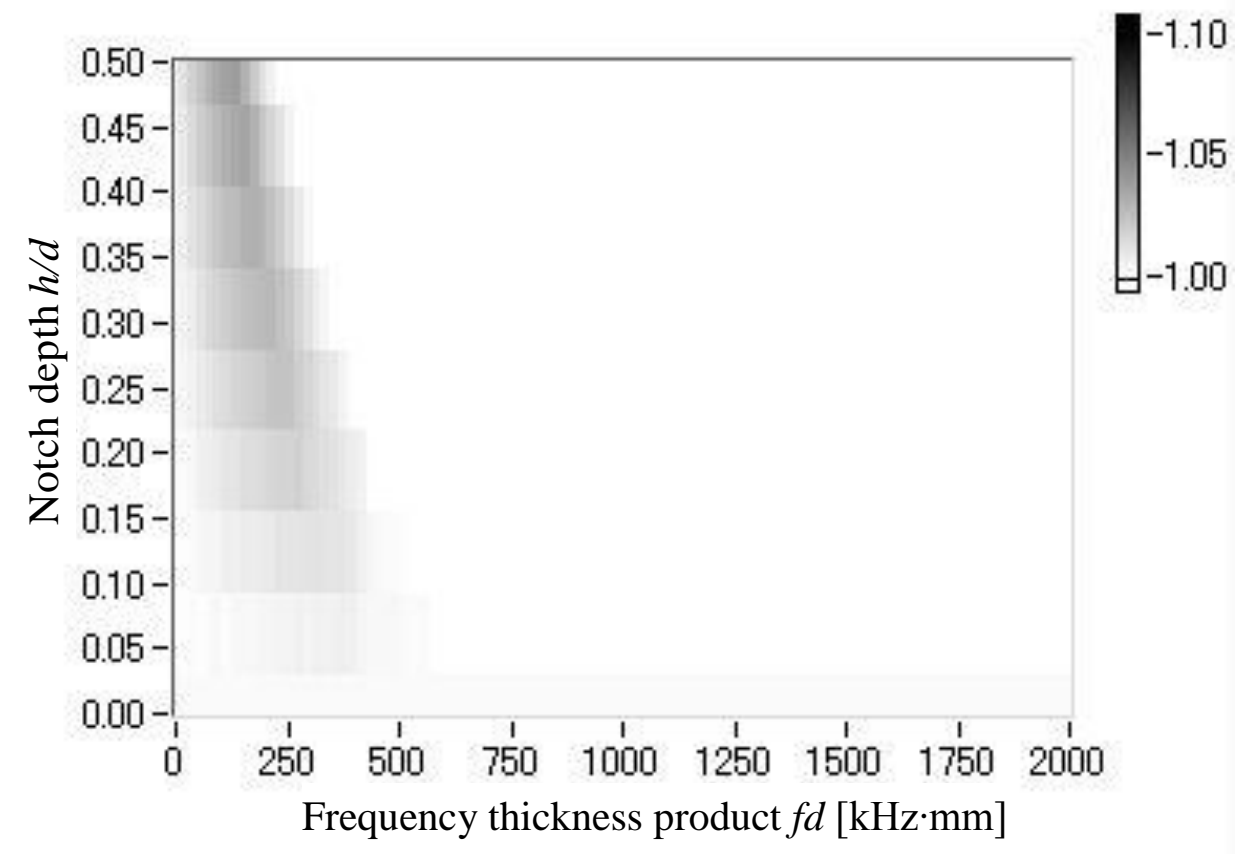

(a) $w / d=0.25$

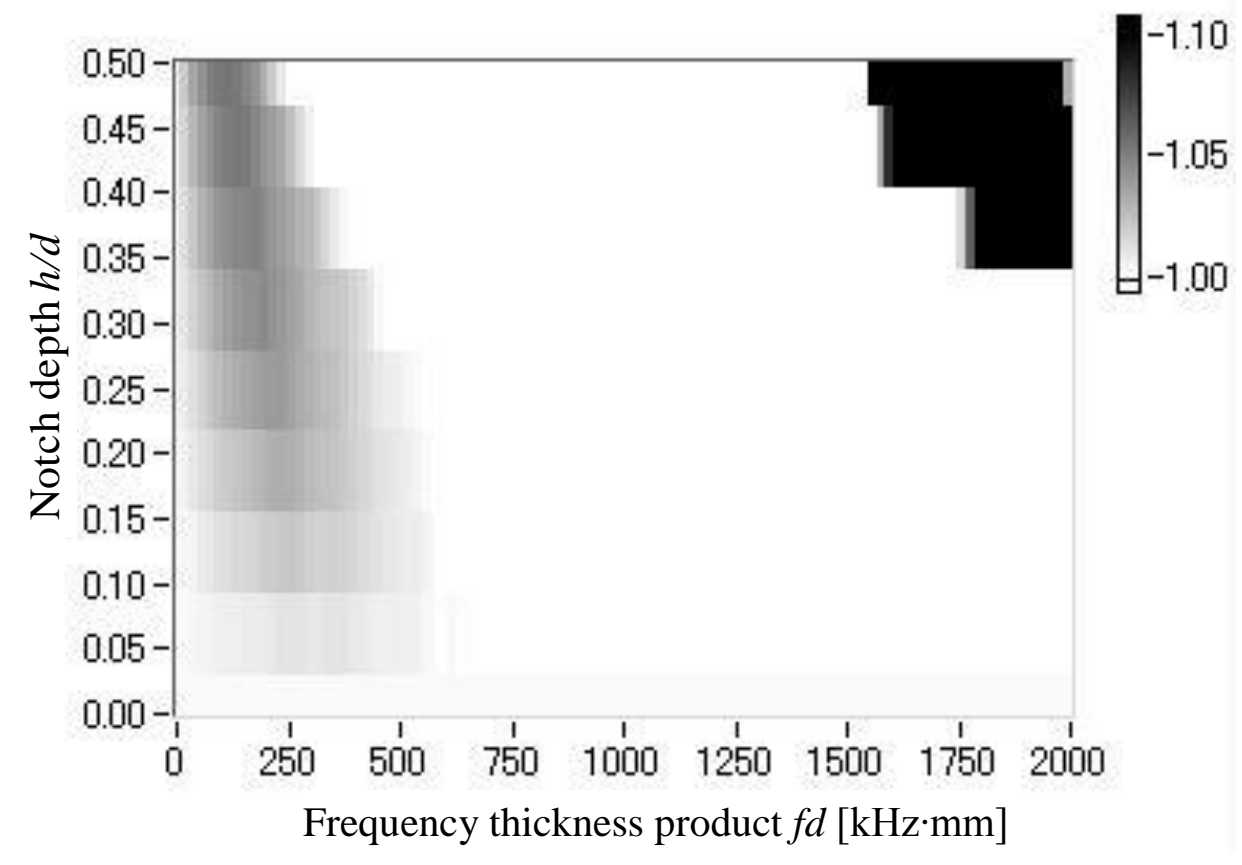

(b) $w / d=0.5$ 


\section{Figure 8}

Experimental set-up for imaging with the scanning laser source technique

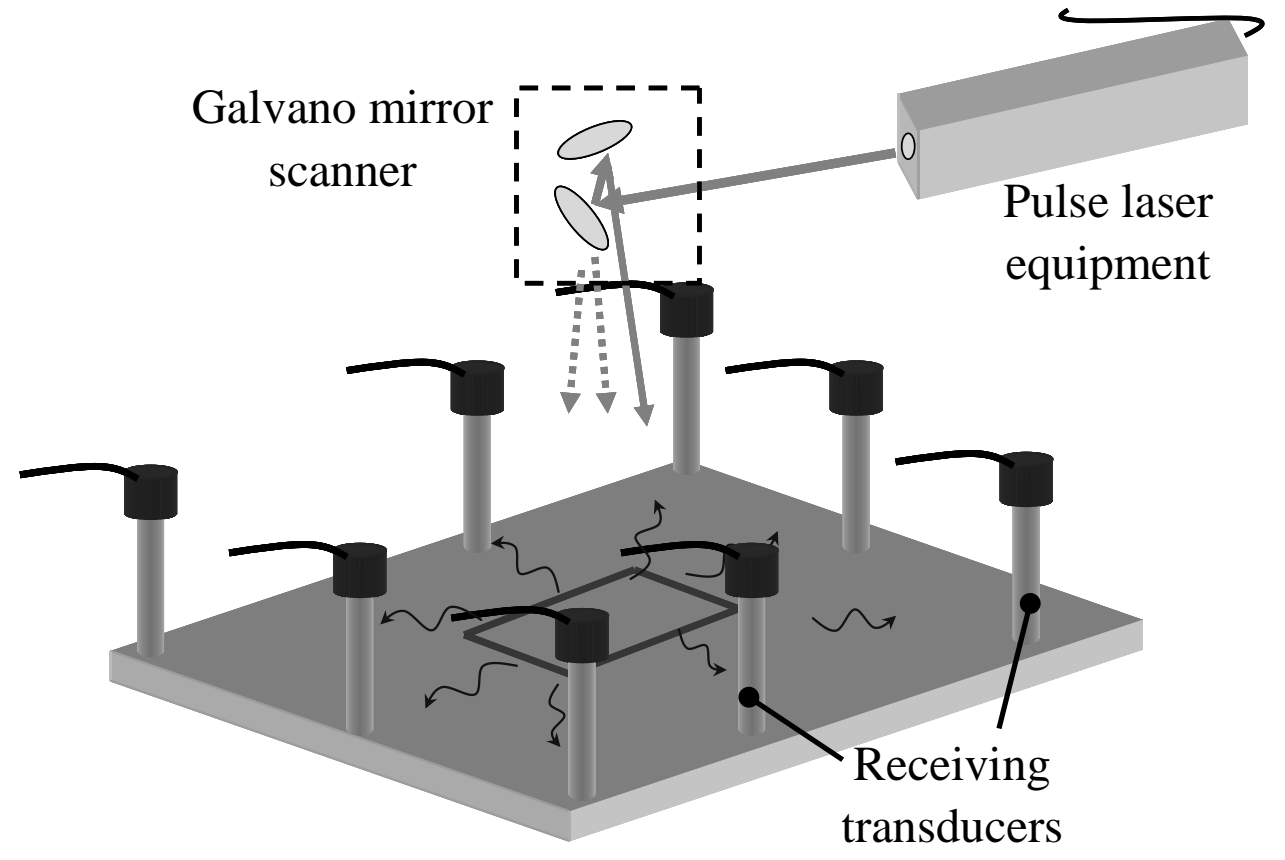


Figure 9

An aluminum plate with notch-type defects used in the experiments
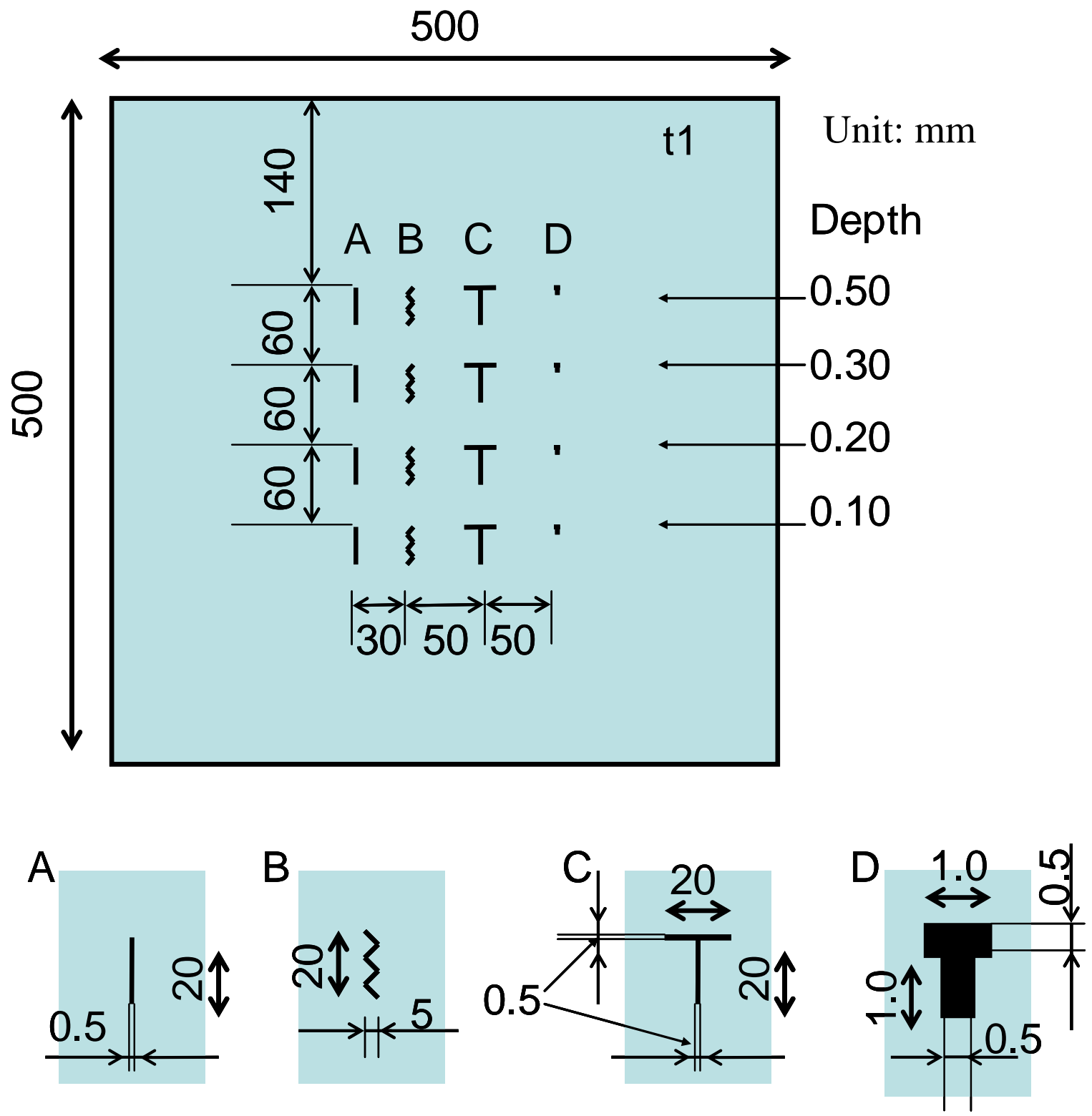
Figure 10

Receiver locations and scanning regions in the experiment for straight notches.

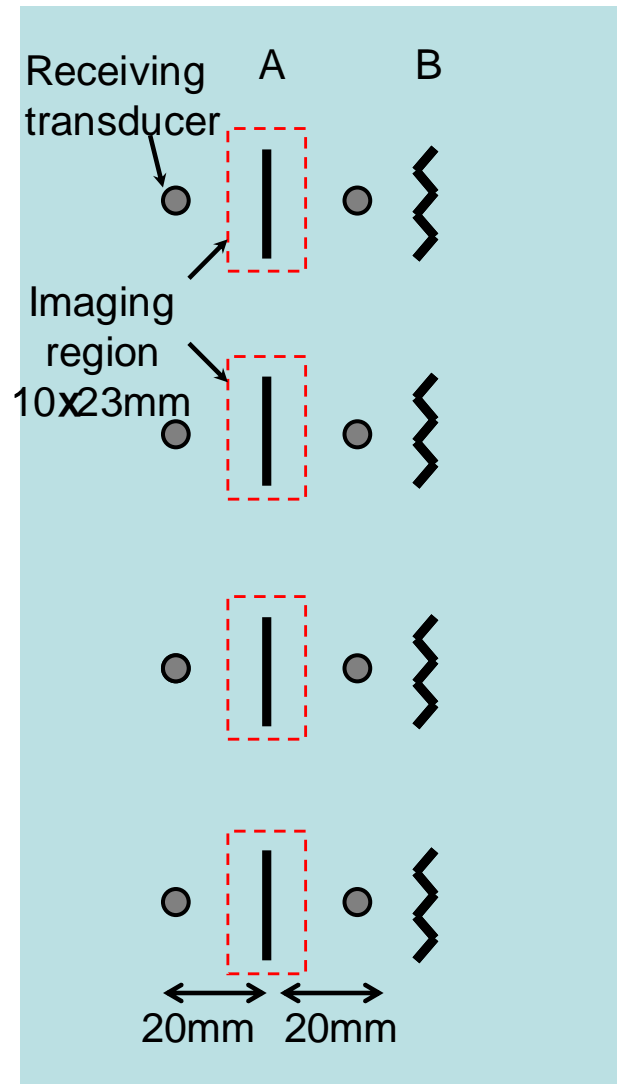




\section{Figure 11}

Defect images of straight notches with four different depths and in 8 different frequency ranges.

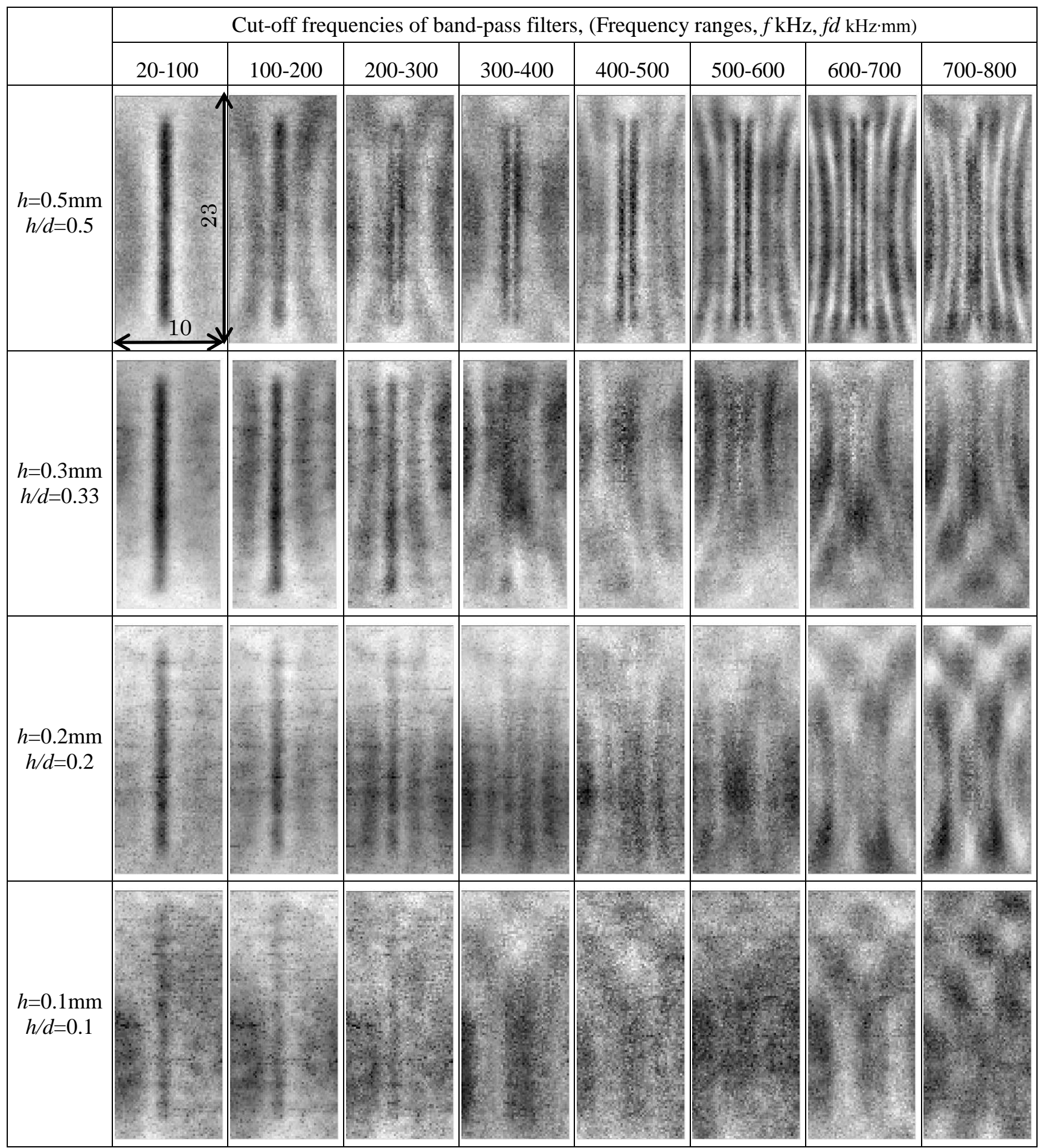


Figure 12 Synthesized images using eight receiving transducers in four frequency ranges for a plate with defects of various shapes.

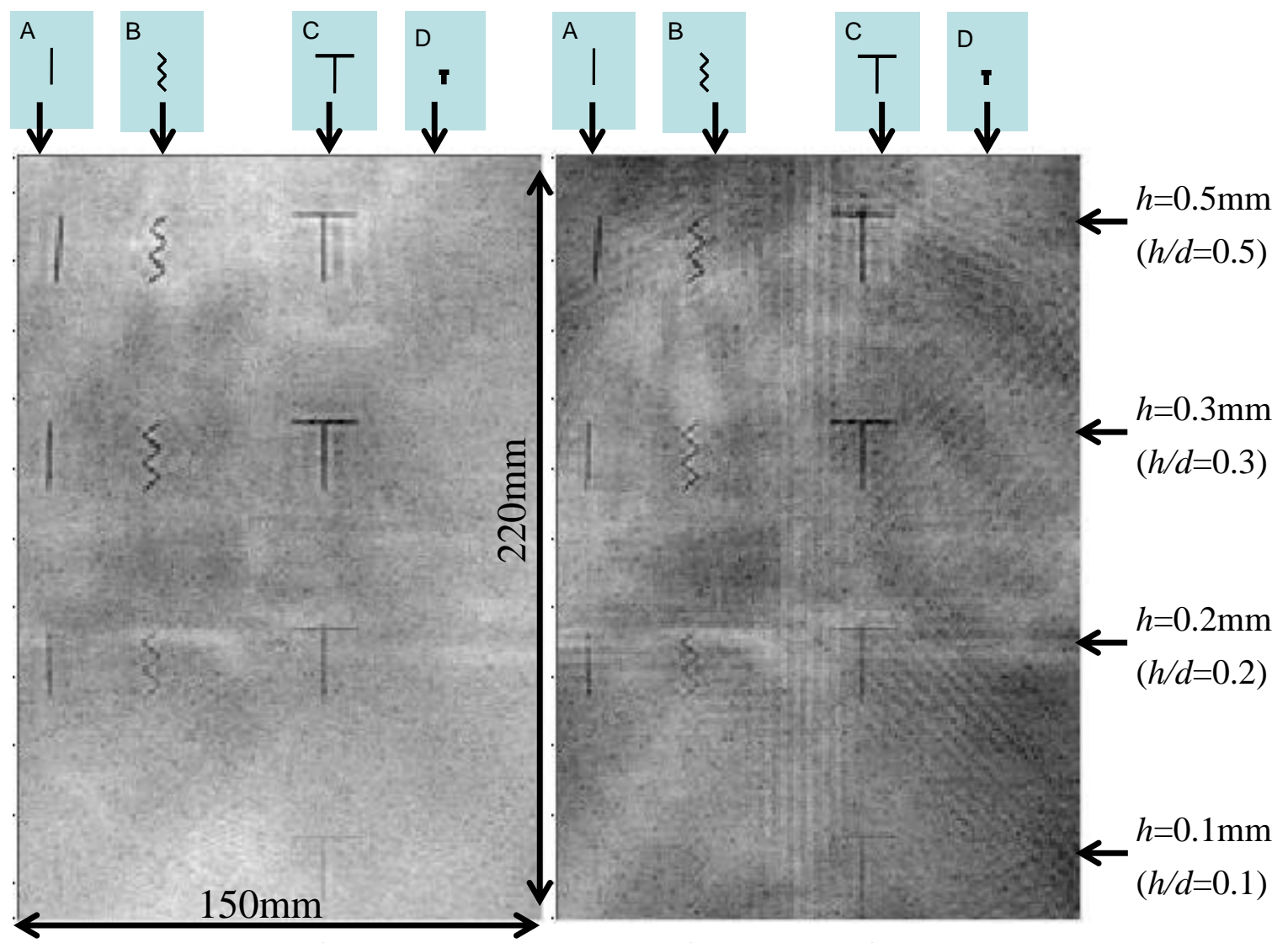

(a) $20-100 \mathrm{kHz}$

(b) $100-200 \mathrm{kHz}$
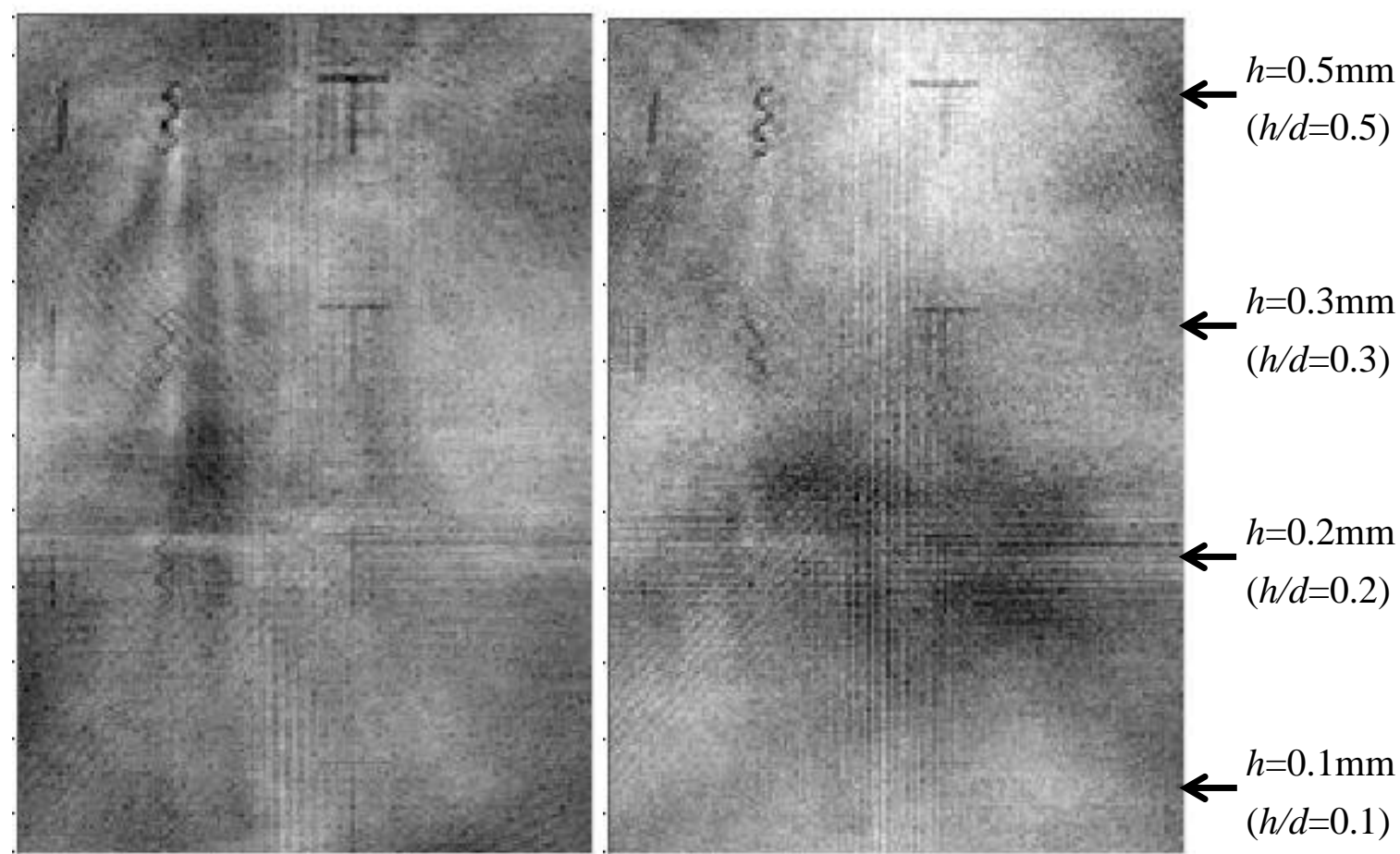

(c) $200-300 \mathrm{kHz}$

(d) $300-400 \mathrm{kHz}$ 


\section{Figure 1}

Schematic figures representing the relationship between location of laser incidence and amplitude of receiving signals.
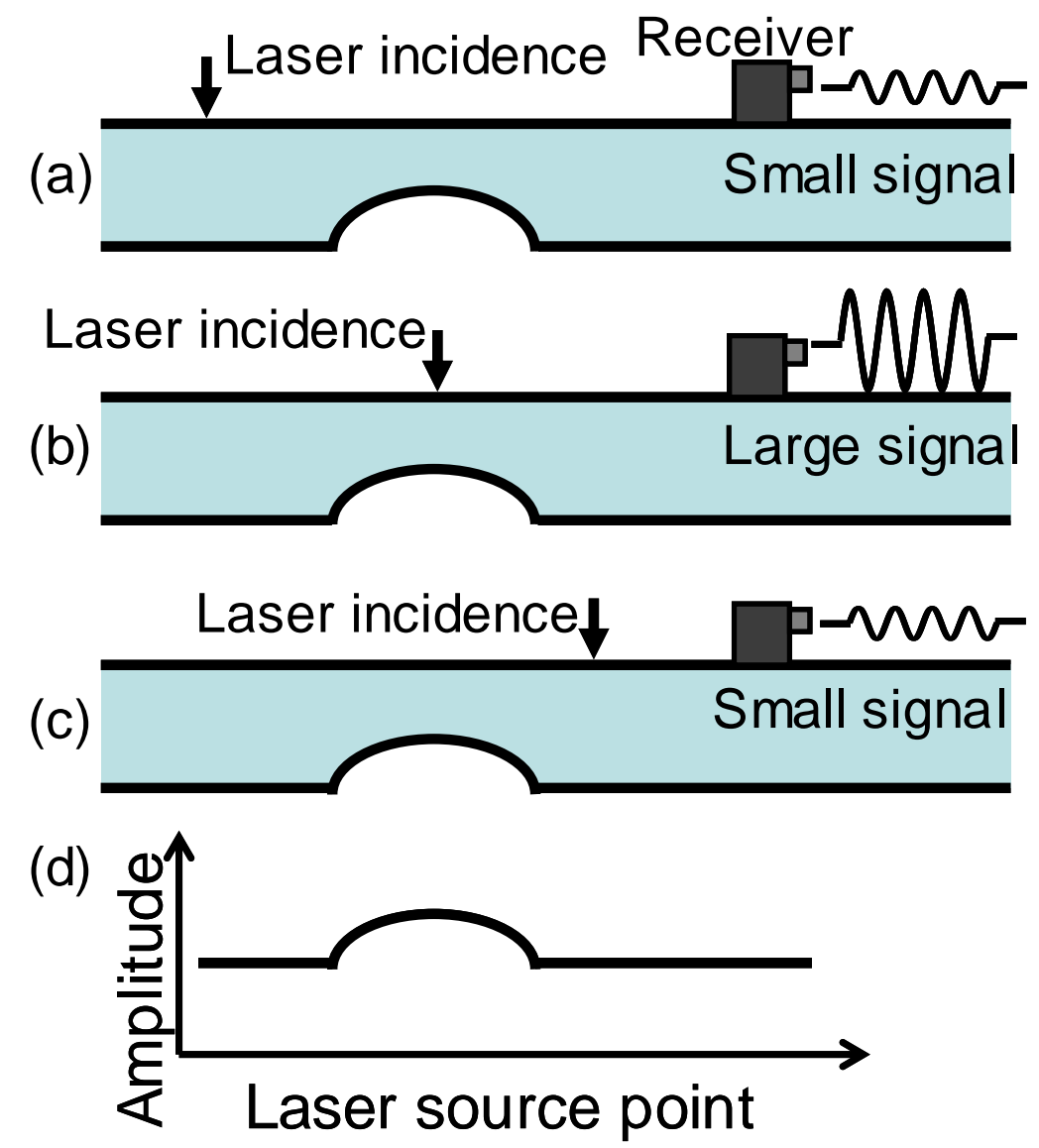
Figure 2

Schematic figures of amplitude distributions affected by large reflections and synthesized distributions using two receiving transducers.

(a)
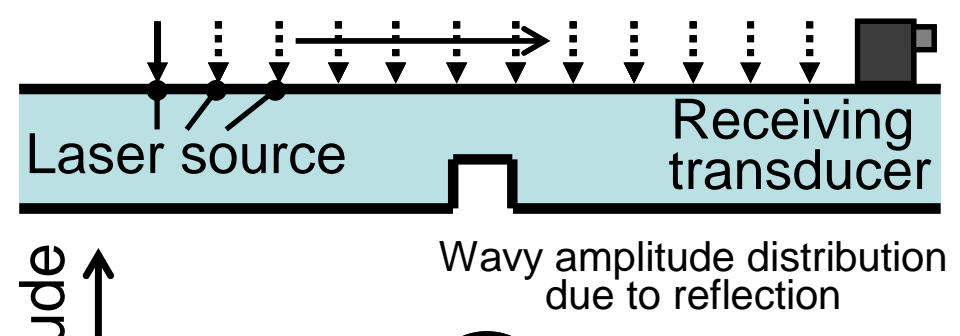

(b)

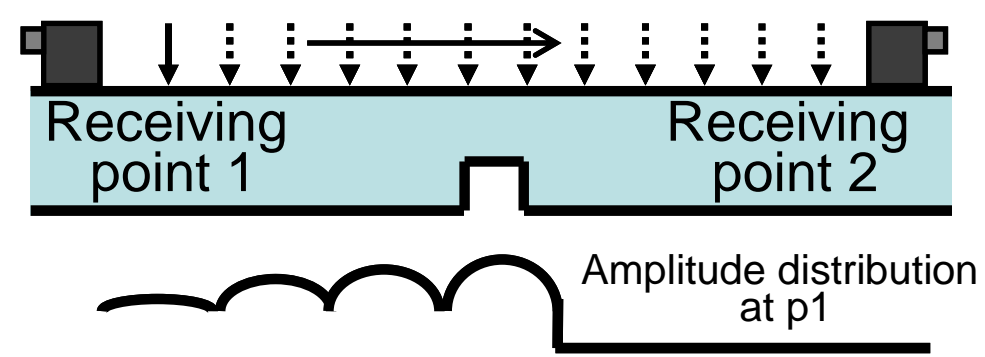

Amplitude distribution at p2

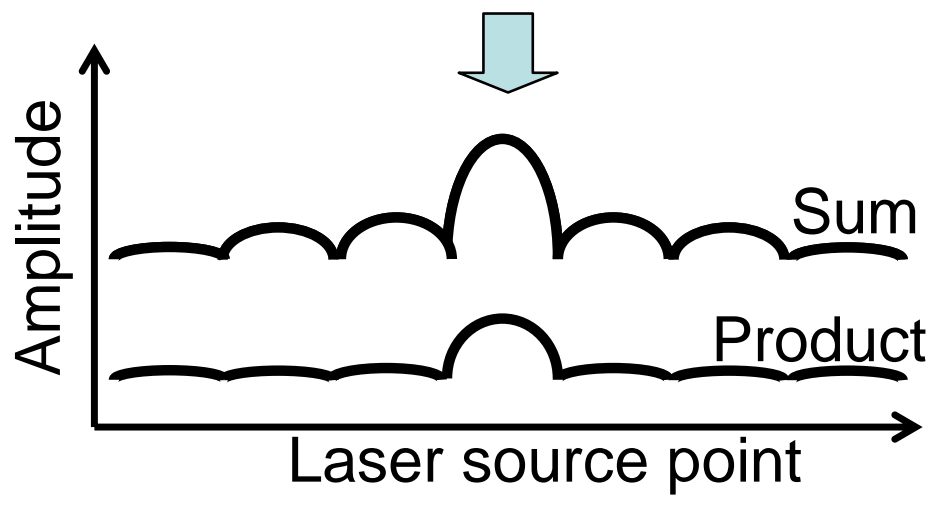




\section{Figure 3}

Combination model of SAFE and FE for calculating amplitude distributions.

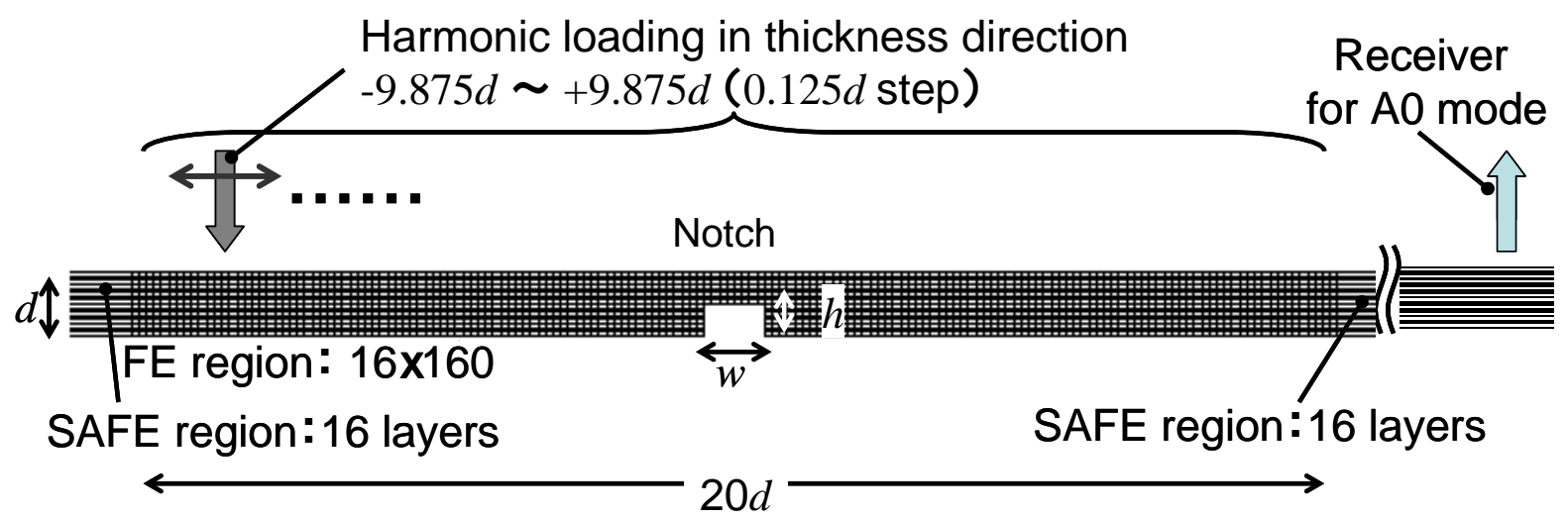


Figure 4

Amplitude distributions with a single receiver around a notch of $w / d=0.5$ and $h / d=0.5$ for various $f d$.

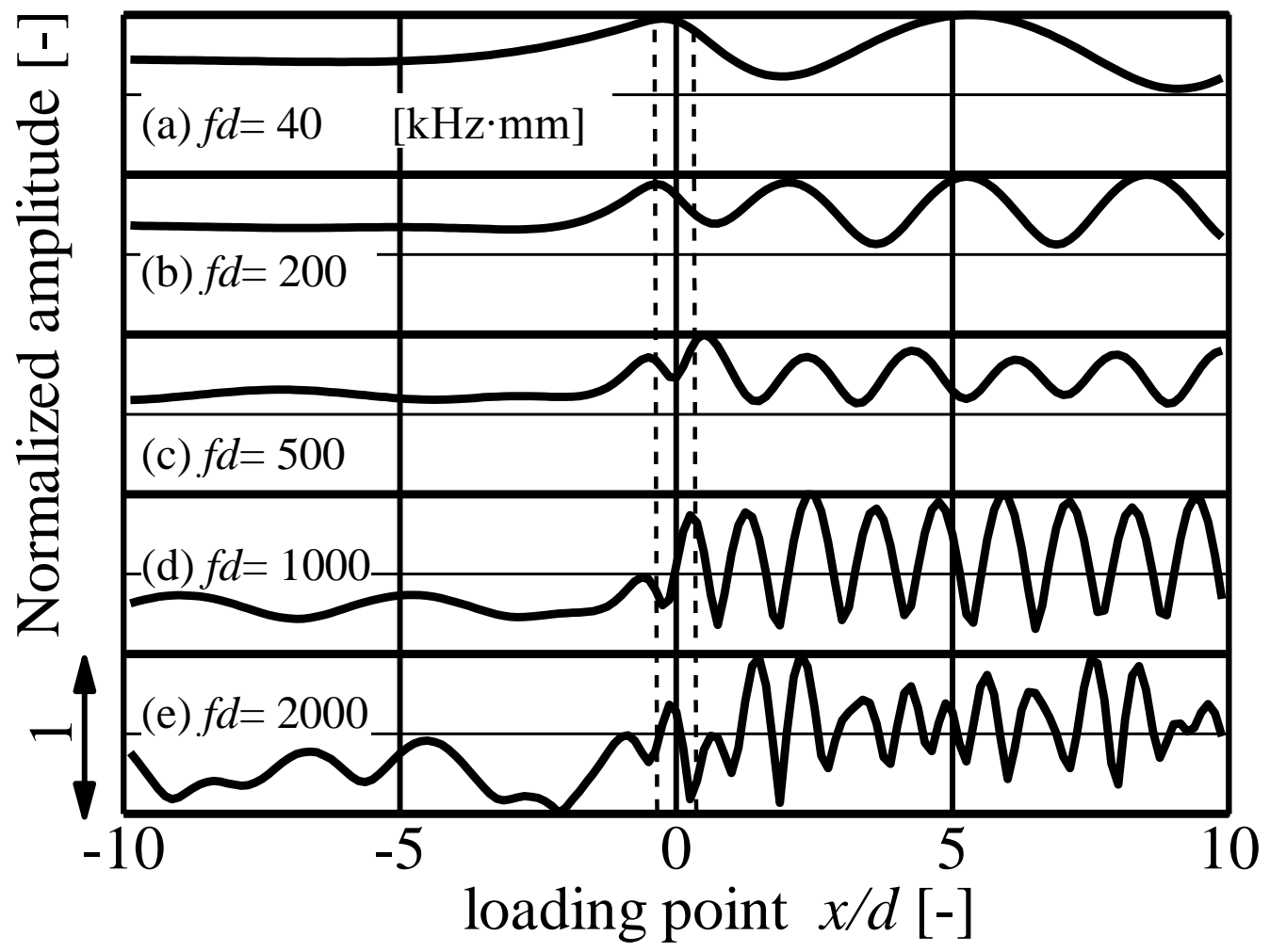


Figure 5

Summations of amplitude distributions with dual receivers located at both sides of a notch with $w / d=0.5$ and $h / d=0.5$ for various $f d$.

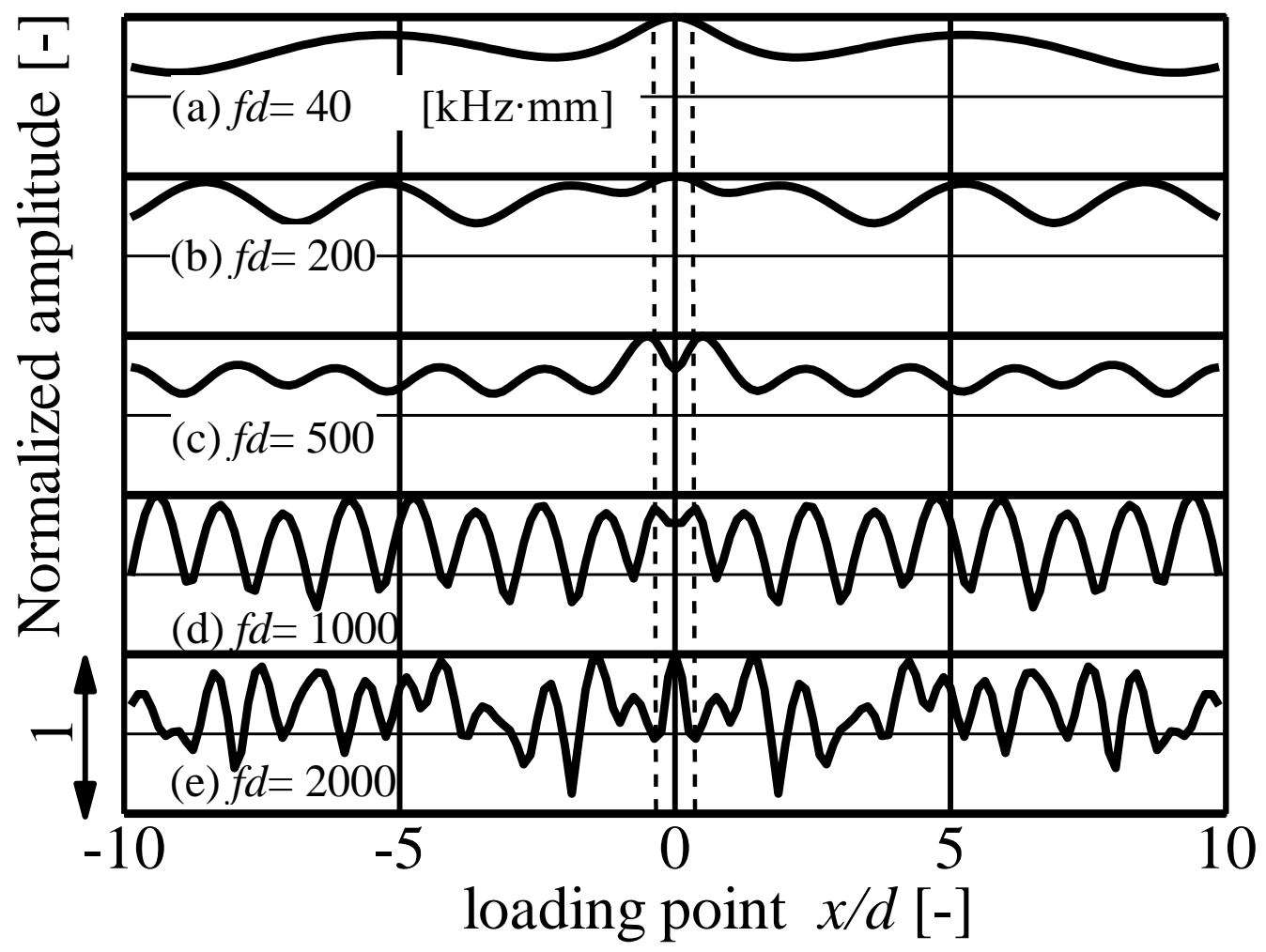


Figure 6

Definition of the imaging performance index $A / B$.

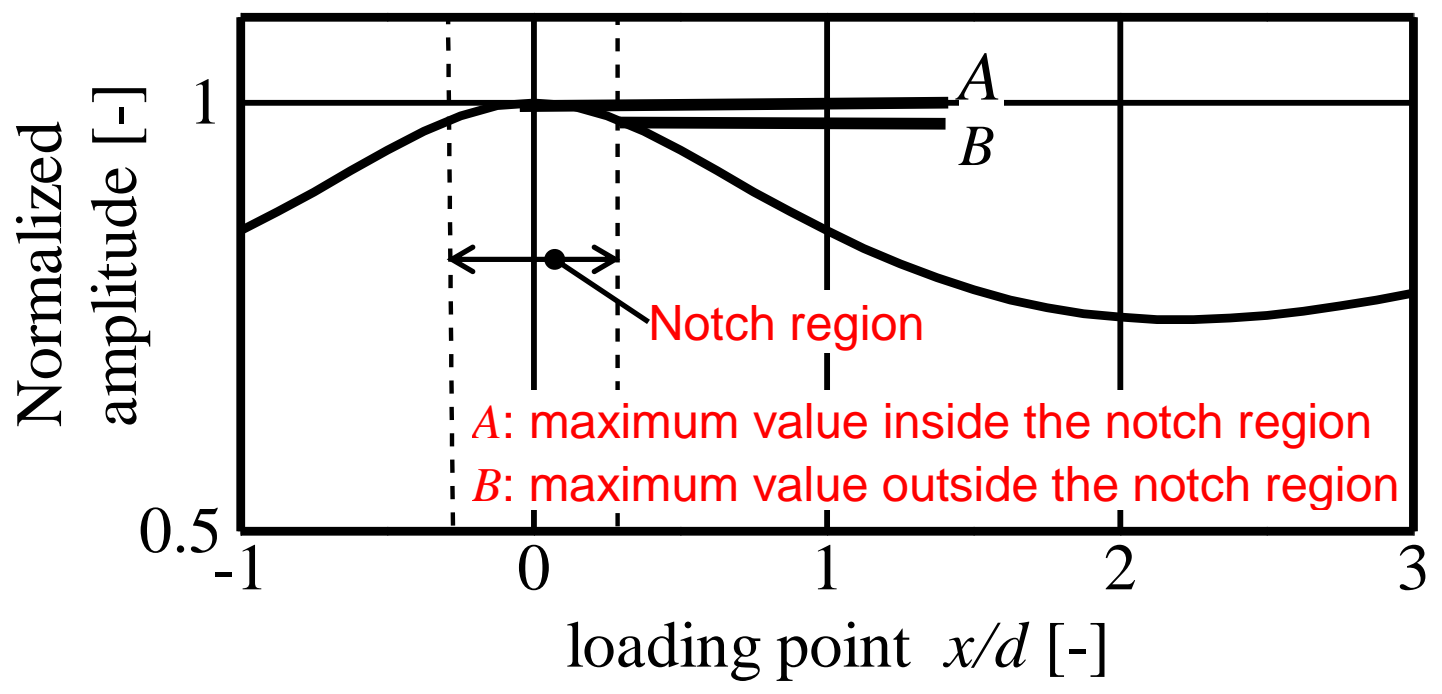




\section{Figure 7}

Frequency dependence of the imaging performance index $A / B$.

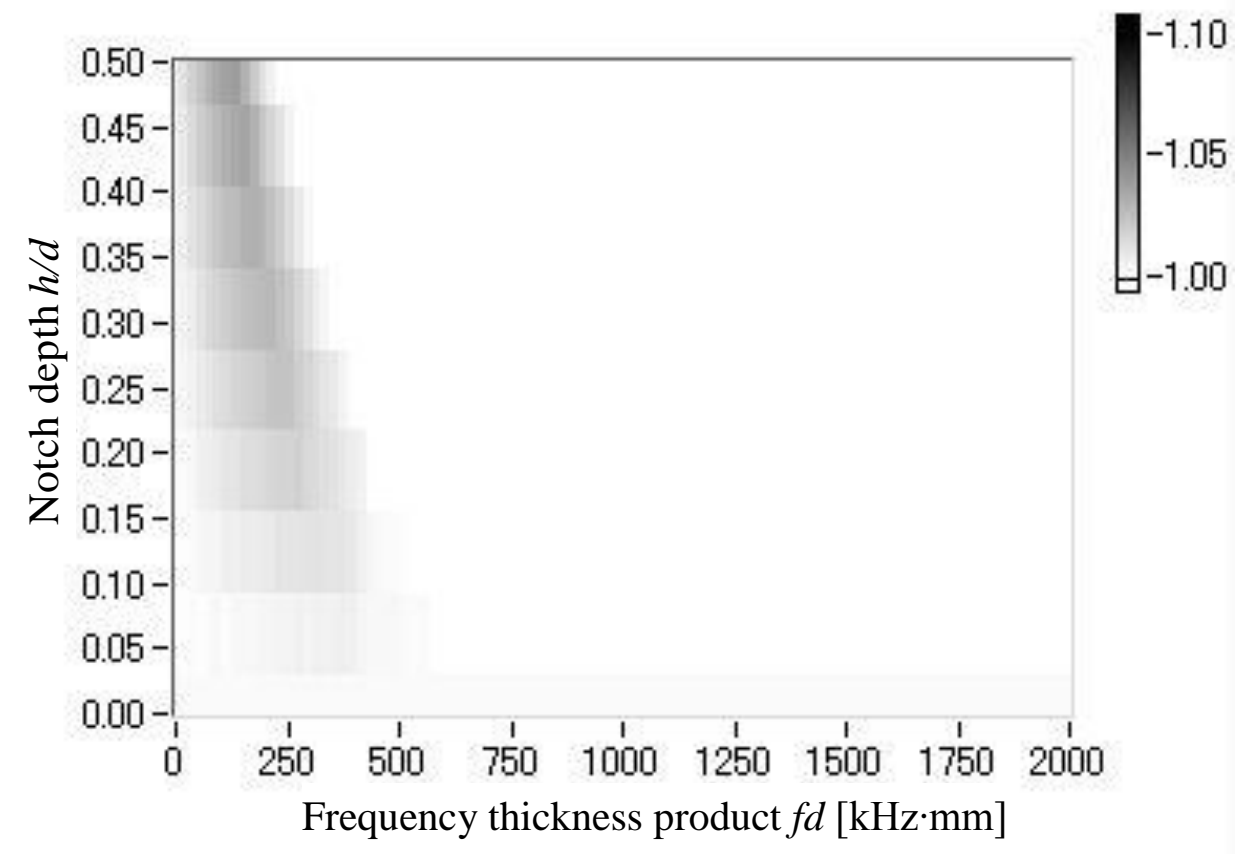

(a) $w / d=0.25$

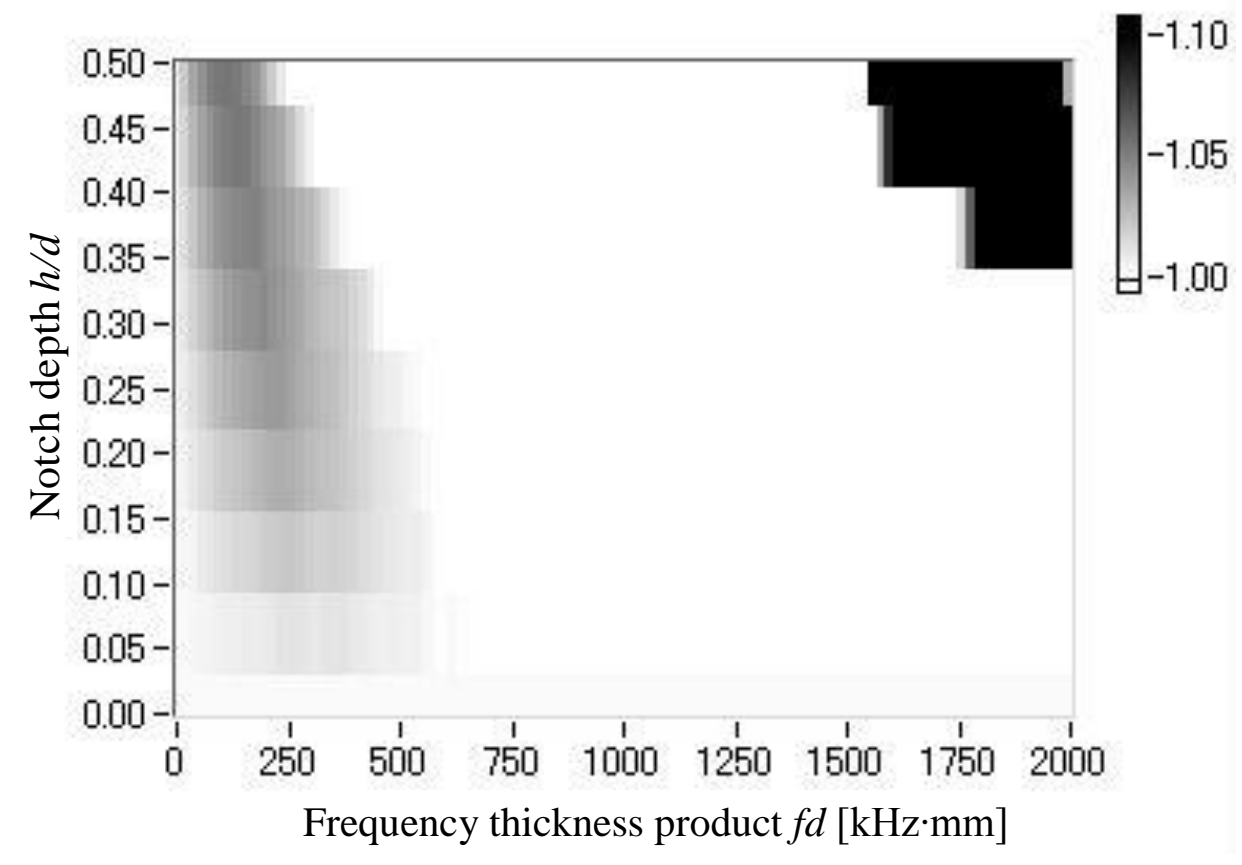

(b) $w / d=0.5$ 


\section{Figure 8}

Experimental set-up for imaging with the scanning laser source technique

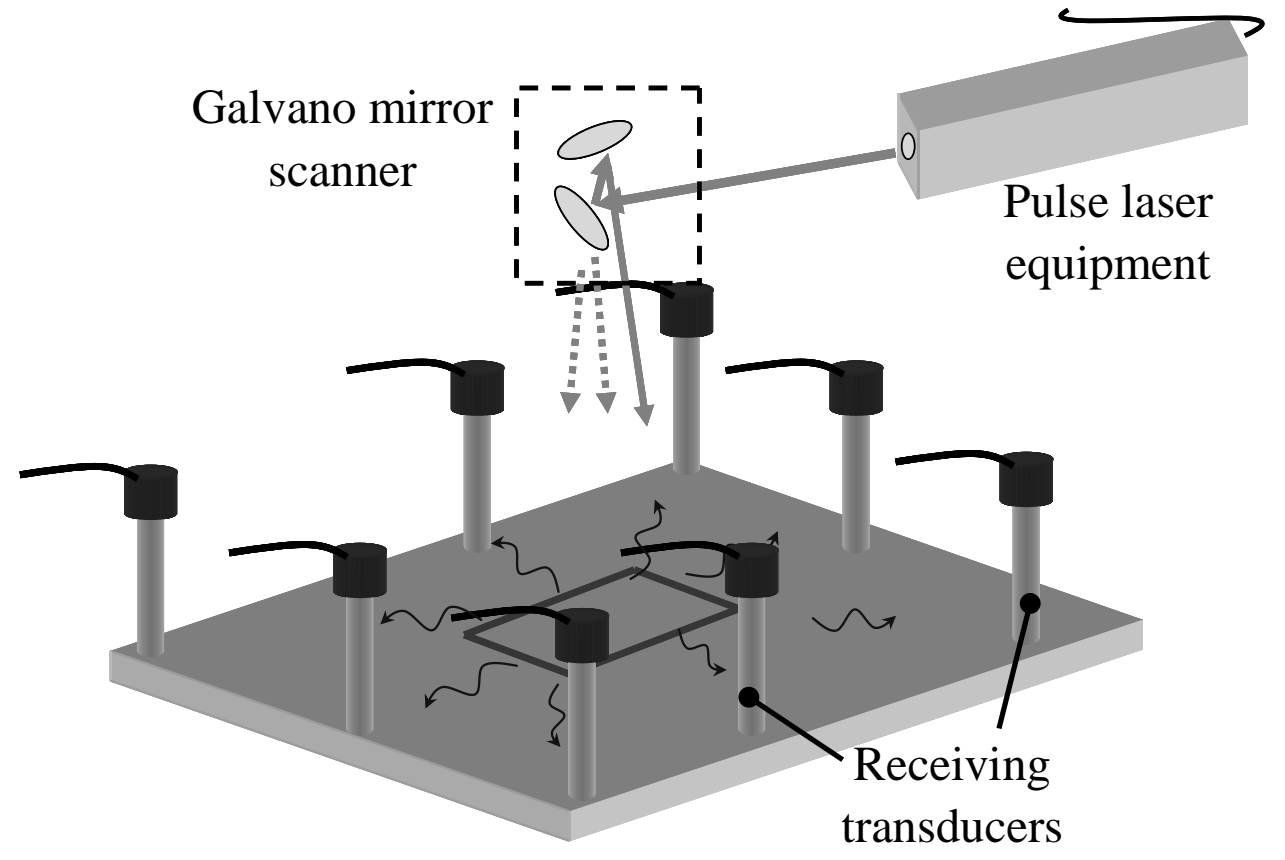


Figure 9

An aluminum plate with notch-type defects used in the experiments
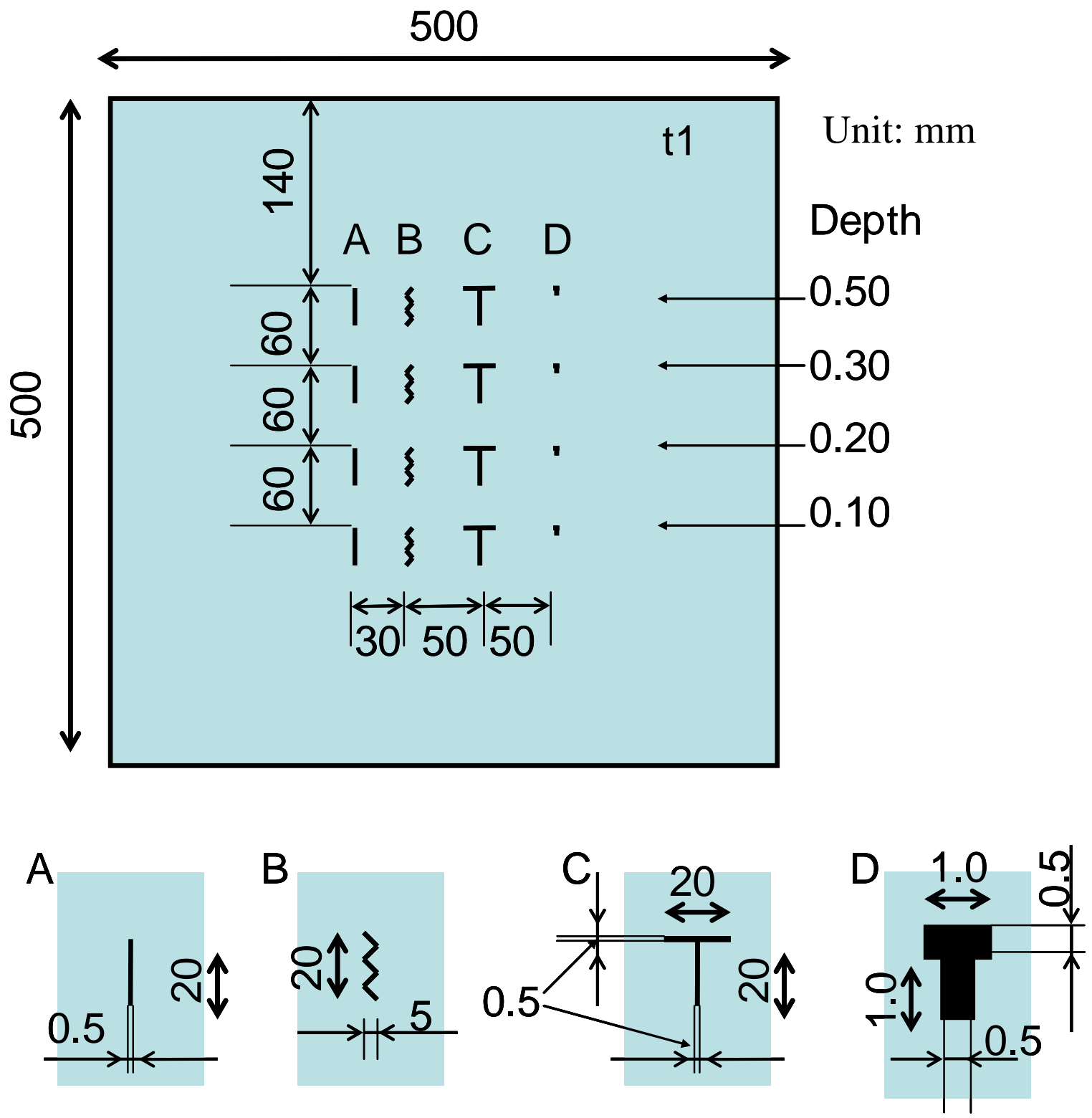
Figure 10

Receiver locations and scanning regions in the experiment for straight notches.

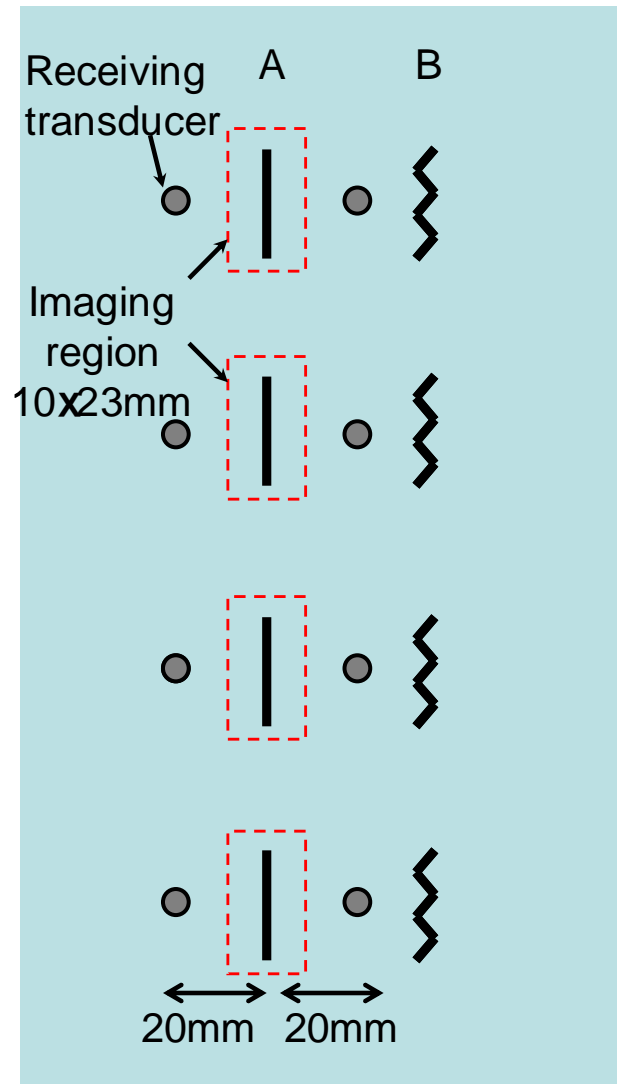




\section{Figure 11}

Defect images of straight notches with four different depths and in 8 different frequency ranges.

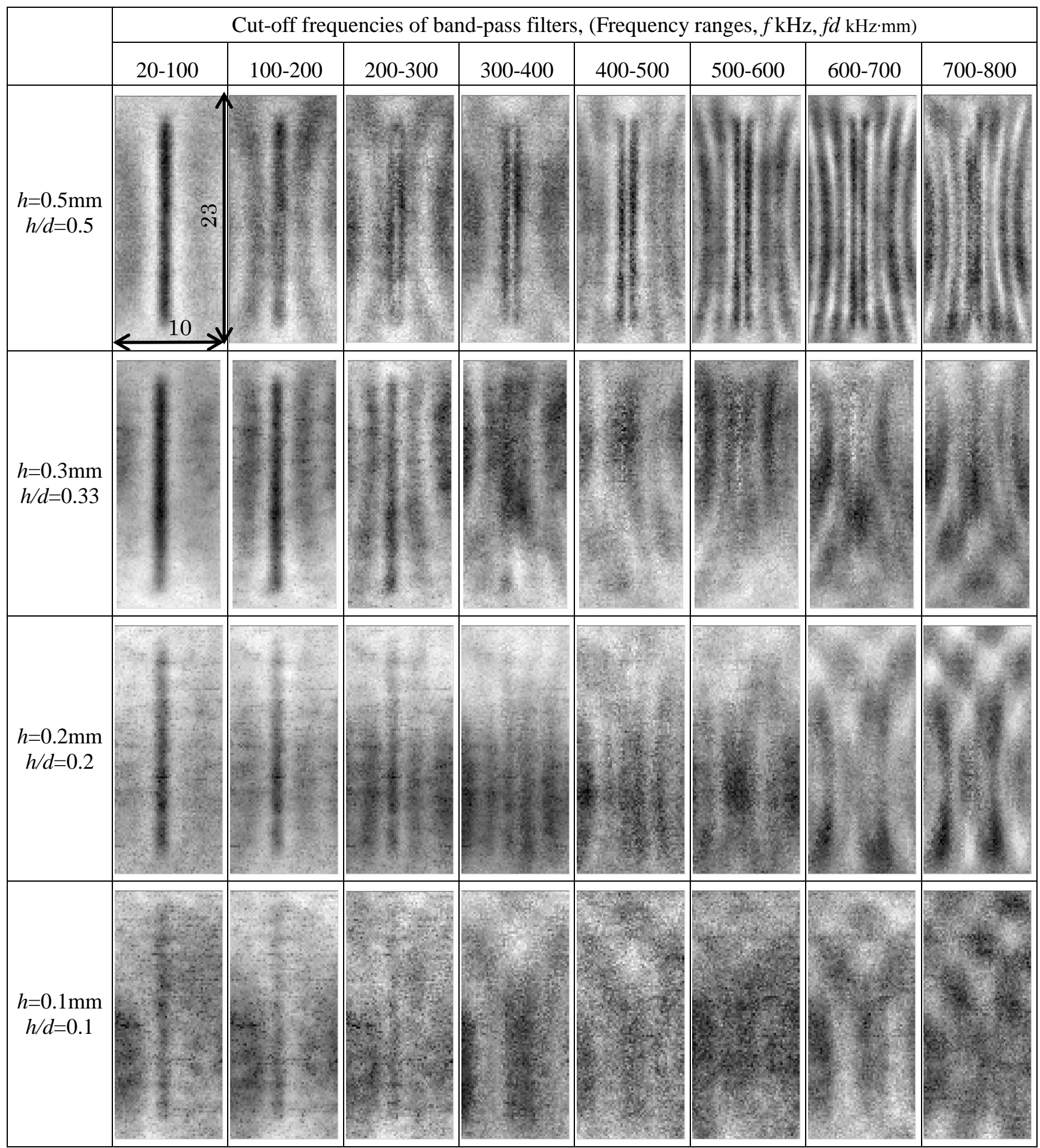


Figure 12 Synthesized images using eight receiving transducers in four frequency ranges for a plate with defects of various shapes.

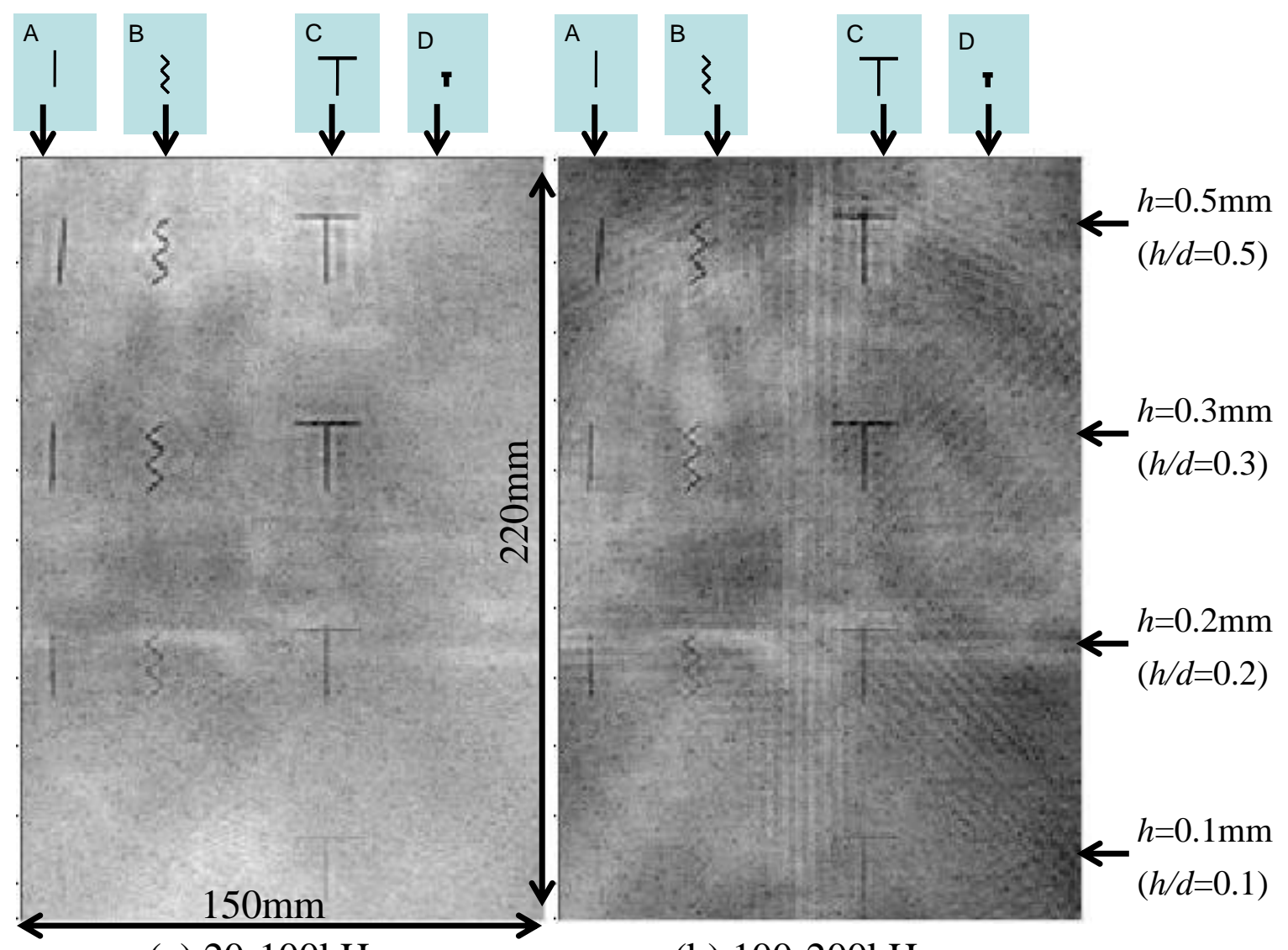

(a) $20-100 \mathrm{kHz}$

(b) $100-200 \mathrm{kHz}$
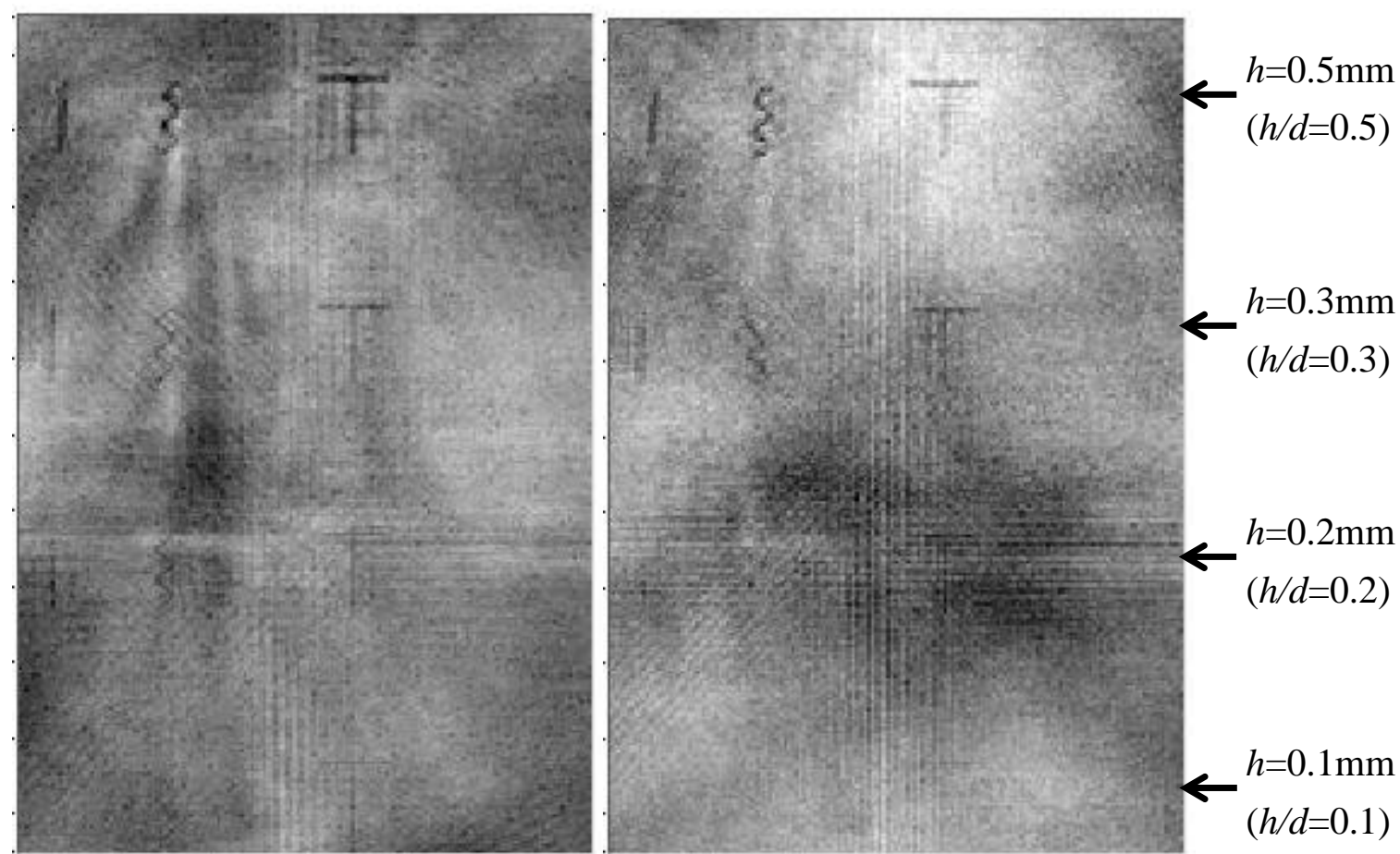

(c) $200-300 \mathrm{kHz}$

(d) $300-400 \mathrm{kHz}$ 
We examine frequency dependence of defect images in scanning laser source technique for a plate. The best frequency range for clear images was obtained by both calculations and experiments. It resulted in the range of frequency-thickness product below about $200 \mathrm{kHz} \cdot \mathrm{mm}$. 